\title{
Copyright
}

by

Michael Skylar Lawson

2011 
The Thesis Committee for Michael Skylar Lawson Certifies that this is the approved version of the following thesis:

\section{Measurement of Deformation of Rotating Blades Using Digital Image Correlation}

\section{APPROVED BY \\ SUPERVISING COMMITTEE:}

Supervisor:

Jayant Sirohi

Krishnaswamy Ravi-Chandar 


\title{
Measurement of Deformation of Rotating Blades Using Digital Image Correlation
}

by

Michael Skylar Lawson, B.S.As.E.

\author{
Thesis \\ Presented to the Faculty of the Graduate School of \\ The University of Texas at Austin \\ in Partial Fulfillment \\ of the Requirements \\ for the Degree of
}

Master of Science in Engineering

The University of Texas at Austin

August 2011 


\section{Acknowledgements}

I would like to show my gratitude to my advisor, Dr. Jayant Sirohi, for the support and guidance he showed throughout writing my thesis and my overall graduate education; it would not have been possible without his help. I would also like to thank Richard Prevost and LaVision for their continued assistance with the digital image correlation software.

Furthermore, I am obliged to many of my colleagues who assisted my research, particularly Jérôme Sicard with his work in flexible rotor blades. Finally, I am thankful to my father for the financial support he has provided during my years in school. 


\begin{abstract}
Measurement of Deformation of Rotating Blades Using Digital Image Correlation
\end{abstract}

\author{
Michael Skylar Lawson, M.S.E. \\ The University of Texas at Austin, 2011
}

Supervisor: Jayant Sirohi

An experimental study on the application of Digital Image Correlation (DIC) to measure the deformation and strain of rotating blades is described. Commercial DIC software was used to obtain measurements on three different types of rotors with diameter ranging from $18^{\prime \prime}$ to $39^{\prime \prime}$ and with varying flexibility to explore applicability of the technique over a breadth of scales. The image acquisition was synchronized with the frequency of rotation such that images could be obtained at the same phase and the consistency of measurements was observed. Bending and twist distributions were extracted from the data with deformation as high as $0.4^{\prime \prime}$ measured with a theoretical accuracy of $0.0038^{\prime \prime}$ and span-wise resolution of $0.066^{\prime \prime}$. The technique was demonstrated to have many advantages including full-field high resolution results, nonintrusive measurement, and good accuracy over a range of scales. The span-wise deformation profiles from the DIC technique are used in conjunction with Blade Element Momentum Theory to calculate the thrust and power consumed by the rotor with rigid 
blades; results are comparable to load cell measurements albeit thrust is somewhat underpredicted and power is over-predicted. Overall, the correlation between DIC calculated thrust and BEMT approximations for comparable blades with constant pitch were within $12 \%$ through the onset of stall. Measurement of flexible blade deformation that would not have been possible with other techniques demonstrated the utility of the DIC method and helped to confirm predictions of flexible blade behavior. 


\section{Table of Contents}

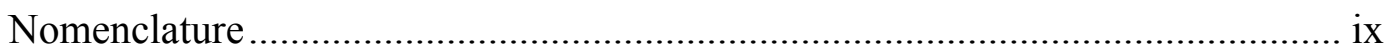

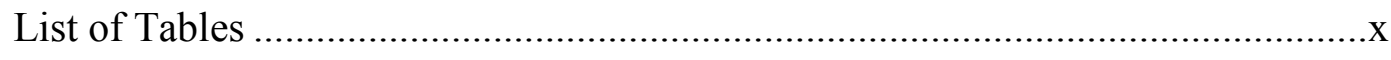

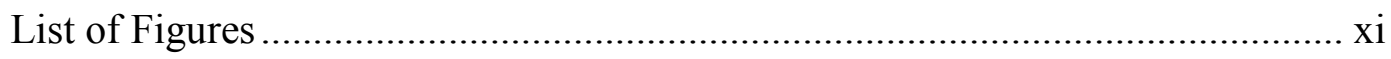

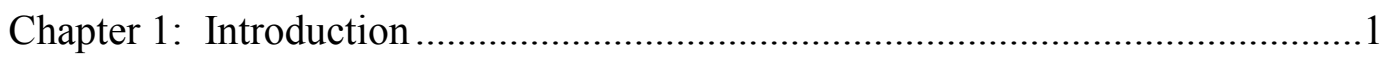

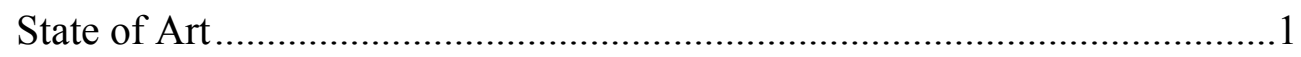

Blade Deformation Measurement .........................................................

Digital Image Correlation ....................................................................

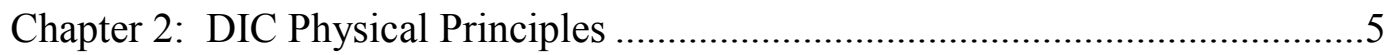

Digital Image Correlation technique...........................................................

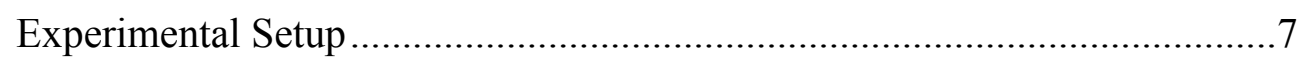

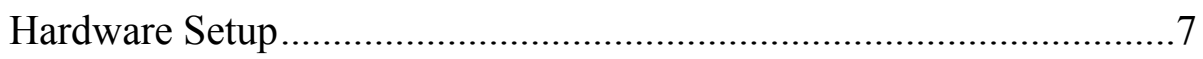

Test Article Preparation .................................................................

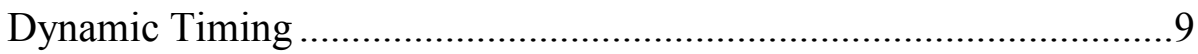

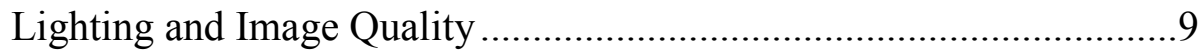

Experiment Procedure.............................................................................. 10

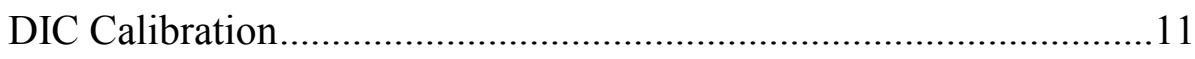

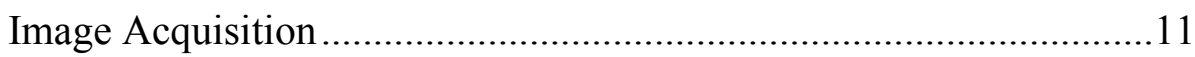

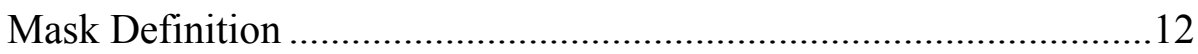

Surface Height Calculation ..................................................................13

Deformation Vector Field Computation ..............................................15

Error Analysis of DIC Measurements.............................................................16

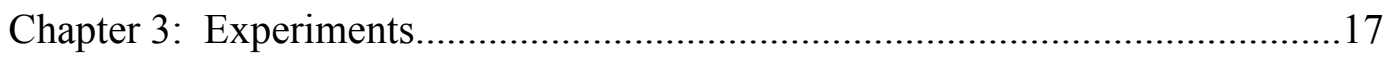

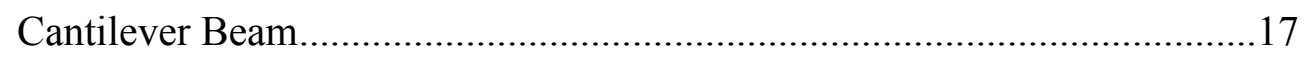

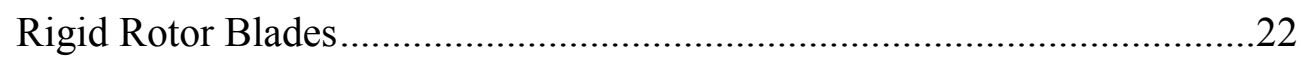

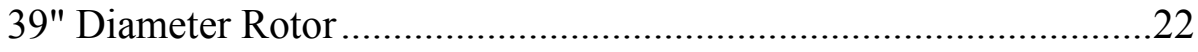

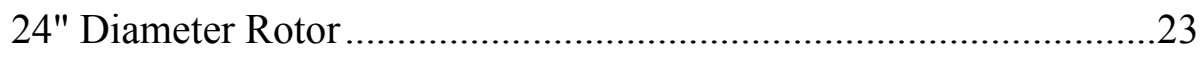

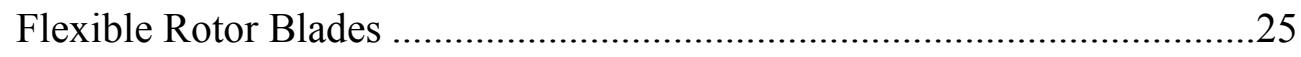




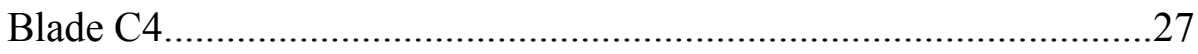

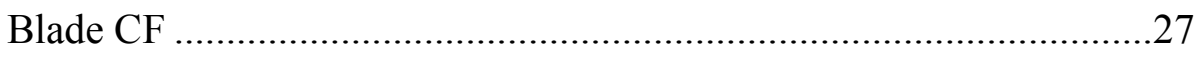

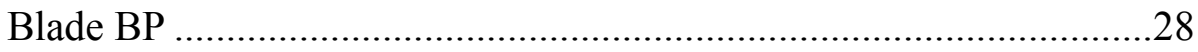

Chapter 4: Performance Analysis using Blade Element Momentum Theory .......29

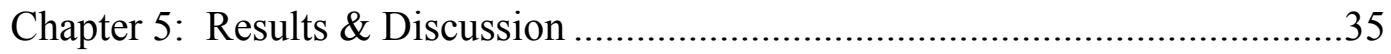

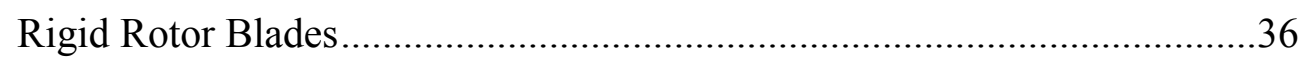

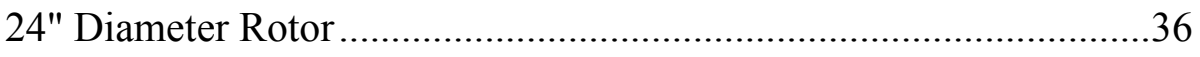

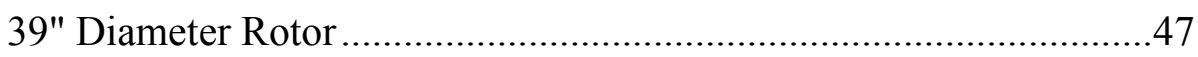

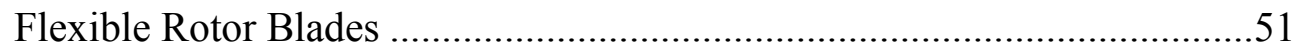

Baseline Blade C4 .......................................................................52

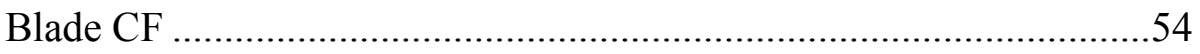

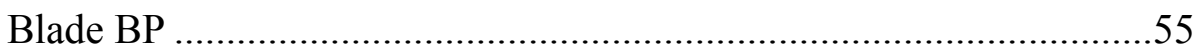

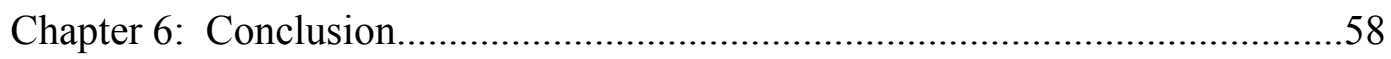

Appendix

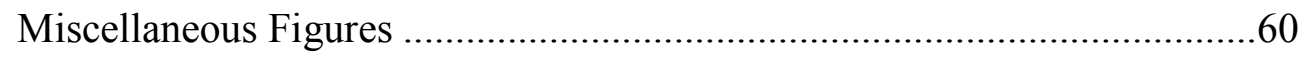

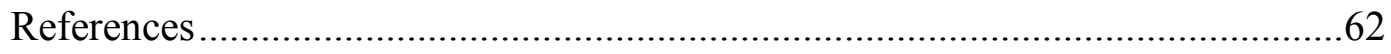

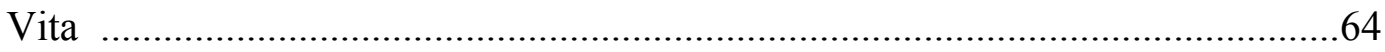




\section{Nomenclature}

$\begin{array}{lll}D I C & = & \text { Digital Image Correlation } \\ L D S & = & \text { Laser Displacement Sensor } \\ M A V & = & \text { Micro Air Vehicle } \\ B E M T & = & \text { Blade Element Momentum Theorem } \\ \varepsilon_{x x} & = & \text { longitudinal strain } \\ z & = & \text { thickness coordinate from neutral axis } \\ w & = & \text { deflection } \\ w^{\prime \prime} & = & \text { curvature } \\ x, r & = & \text { span-wise coordinate } \\ R & = & \text { length of beam/blade } \\ c & = & \text { length of local chord } \\ g & = & \text { vector grid (distance between pixels) } \\ \Delta t & = & \text { time shift } \\ \theta_{c} & = & \text { collective pitch angle (degrees) } \\ \theta & = & \text { pitch angle (degrees) } \\ \beta_{0}= & \text { rigid flap (coning) angle } \\ C_{T} & = & \text { coefficient of thrust } \\ C_{l a} & = & \text { 2D airfoil section coefficient of lift } \\ C_{P}= & \text { coefficient of power } \\ F M & = & \text { Figure of Merit } \\ T & = & \text { Thrust } \\ v_{i} & = & \text { local incident velocity } \\ \lambda & = & \text { inflow ratio } \\ \sigma & = & \text { rotor solidity } \\ F & = & \text { Prandtl's tip loss correction factor }\end{array}$




\section{List of Tables}

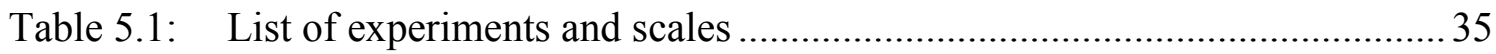

Table 5.2: Variation of thrust and power coefficients, and figure of merit, with collective pitch for $39 "$ diameter rigid rotor ................................... 50

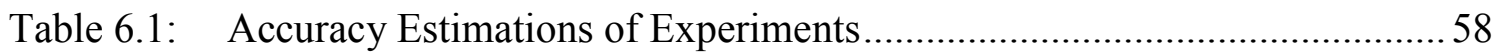




\section{List of Figures}

Figure 1.1: $\quad$ Strain gage mounted on a 0.15 scale Bell 412 helicopter blade ..................

Figure 2.1: Close-up of example speckle pattern ....................................................

Figure 2.2: Typical stereoscopic DIC setup ........................................................6

Figure 2.3: Application of a stochastic speckle pattern via the toothbrush method.......9

Figure 2.4: Example of image masking; provides a clear boundary between background noise and test article speckle pattern ................................13

Figure 2.5: Surface Height Calculation ............................................................. 14

Figure 2.6: Deformation vector field calculation ................................................... 15

Figure 3.1.1: Experimental setup for measuring the deflection of a vibrating cantilever beam using DIC ............................................................ 18

Figure 3.1.2: Schematic for vibrating cantilever beam experiment. ...........................19

Figure 3.1.3: Cantilever beam deflection measurements from DIC and LDS ..............20

Figure 3.1.4: Deflection vs. span, comparison between raw data and reconstructed curve ..................................................................... 21

Figure 3.1.5: Strain vs. span for vibrating cantilever beam.......................................21

Figure 3.2.1: Experimental setup on the measurement of deformation on 39" diameter rigid rotor blades using DIC ............................................23

Figure 3.3.1: 24" rigid rotor blades on the hover test stand .....................................24

Figure 3.3.2: Experimental setup on the measurement of deformation on 24" diameter rigid rotor blades using DIC .24

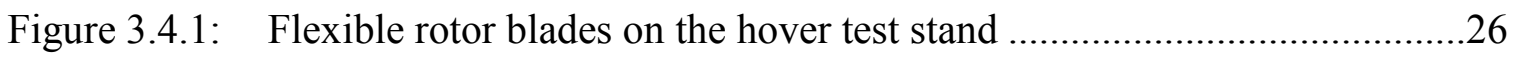

Figure 3.4.2: Comparison between rotor blades used in experiments..........................26

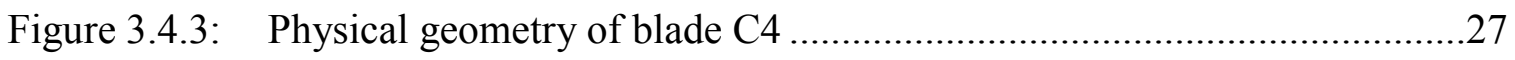




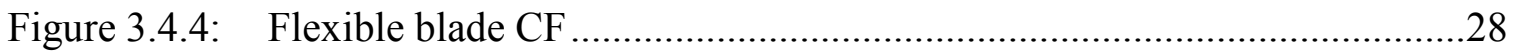

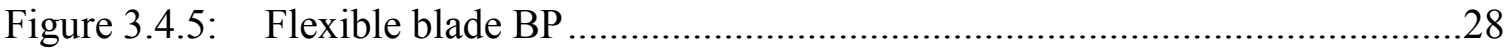

Figure 4.1: Simplified flow model of momentum theory analysis of a rotor in hover....29

Figure 4.2: Aerodynamic forces and moments acting on a typical blade element; illustration of blade physical characteristics. .........................................30

Figure 4.3: $\quad$ Flow chart illustrating BEMT processing of DIC data .................................

Figure 5.1.1: Raw deflection data for all images in a set for 24" diameter rigid blades; $1800 \mathrm{rpm}$ at 8.5 degree collective pitch and mean deflection with error bars

Figure 5.1.2: $\quad$ Illustration for the method of sectional pitch calculation 38

Figure 5.1.3: Difference between a typical and an off-horizontal data set illustrating the change in measured chord length

Figure 5.1.4: Raw pitch distribution data for all images in a set for 24" diameter rigid blades; $1800 \mathrm{rpm}$ at 8.5 degree collective pitch and mean pitch distribution with error bars

Figure 5.1.5: Mean deflection curves for 24" diameter rigid blades at $1500 \mathrm{rpm}$; various collective pitch angles

Figure 5.1.6: Mean pitch distribution curves for 24" diameter rigid blades at 1500 rpm; various input collective pitch angles

Figure 5.1.7: Mean deflection curves for 24" diameter rigid blades at $1500 \mathrm{rpm}$ and $1800 \mathrm{rpm} ; 6.07^{\circ}$ and $14.76^{\circ}$ collective pitch angles

Figure 5.1.8: Rigid flap angle vs. collective pitch angle for 24" diameter rigid blades at $1500 \mathrm{rpm}$ and $1800 \mathrm{rpm}$.

Figure 5.1.9: Inclinometer measured collective pitch vs. DIC measured collective pitch for both 1500 and $1800 \mathrm{rpm}$. 
Figure 5.1.10: Thrust vs. collective pitch angle, comparison between DIC + BEMT results and load cell measurements.

Figure 5.1.11: Thrust coefficient vs. collective pitch angle, comparison between DIC

+ BEMT results and load cell measurements.

Figure 5.1.12: Power coefficient vs. collective pitch angle, comparison between DIC

+ BEMT results and load cell measurements.

Figure 5.1.13: Thrust coefficient vs. power coefficient, comparison between DIC + BEMT results and load cell measurements .46

Figure 5.1.14: Figure of Merit vs. collective pitch angle, comparison between DIC approximation and constant blade element pitch approximation .46

Figure 5.2.1: Mean deflection for 39" diameter rigid blades at 4.5 degree collective ....47

Figure 5.2.2: Mean deflection for 39" diameter rigid blades at 8 degree collective .......48

Figure 5.2.3: Mean twist curves for 39" diameter rigid blades at 4.5 degree collective...49

Figure 5.2.4: Mean twist curves for 39" diameter rigid blades at 8 degree collective ....49

Figure 5.2.5: Rigid flap angle vs. rpm for 39" diameter rigid blades..........................50

Figure 5.2.6: Predicted thrust vs. rpm for 39" diameter rigid blades using span-wise variation in pitch angles measured by DIC in conjunction with BEMT....51

Figure 5.3.1: Thrust vs. collective pitch for each of the flexible blades at 1800rpm ......52

Figure 5.3.2: Mean deflection curves for blade $\mathrm{C} 4$ at various collective pitch..............53

Figure 5.3.3: Mean pitch distribution curves for blade $\mathrm{C} 4$ at various collective ............53

Figure 5.3.4: Mean deflection curves for blade $\mathrm{CF}$ at various collective pitch...............54

Figure 5.3.5: Mean pitch distribution curves for blade $\mathrm{CF}$ at various collective pitch ...55

Figure 5.3.6: Mean deflection curves for blade BP at various collective pitch..............56

Figure 5.3.7: Mean pitch distribution curves for blade BP at various collective pitch ...56

Figure 5.3.8: Mean deflection curves for various blades at $14.76^{\circ}$ collective .57 xiii 
Figure 5.3.9: Mean twist distribution for various blades at $14.76^{\circ}$ collective pitch .......57

Figure A1: $\quad$ Mean deflection curves for 24" diameter rigid blades at $1800 \mathrm{rpm}$;

various collective pitch angles ......................................................60

Figure A2: Airfoil lift coefficient vs. local pitch angle ............................................60

Figure A3: $\quad$ Airfoil drag coefficient vs. local pitch angle .........................................61 


\section{Chapter 1: Introduction}

This thesis describes the use of the stereoscopic three-dimensional digital image correlation (DIC) technique to measure deformation on helicopter rotor blades under rotation. Optical full-field measurement techniques have, over the course of the past few decades, grown increasingly useful in engineering research and development to facilitate design, materials production, and inspection ${ }^{1}$. Holographic Interferometry (HI), Electronic Speckle Pattern Interferometry (ESPI), Moiré techniques, and Digital Image Correlation (DIC) have been thoroughly explored from the theoretical ${ }^{2,3}$ and engineering application $^{4,5}$ viewpoints.

A major advantage of optical techniques is that they are non-contact and do not require extensive instrumentation of the structure. This makes them extremely attractive for the measurement of deformation in rotating systems such as helicopter rotor blades as it eliminates the necessity for bulky electric slip rings. The final results of DIC measurements are comparable to, and can be used to quickly verify, finite element models; this results in a reduction in the number of prototypes required, improving design at lower cost. Additionally, optical techniques do not require involved test article preparation, and can be applied to small or complex geometry that would be otherwise inaccessible to conventional methods.

\section{STATE OF ART}

\section{Blade Deformation Measurement}

Depending on its size and design functions, a helicopter may have a wide range of rotor geometry, operating RPM and tip speed, and have both rigid and elastic flap angles. For example, a micro-air-vehicle (MAV) rotor may be just a few inches in diameter, have 
a chord length under an inch, operational speeds in the thousands of RPM, but relatively low tip velocities on the order of a couple hundred feet per second. On the other end of the spectrum, the Soviet Mil-V12 is the largest helicopter in the world with 2 rotors over 115 feet in diameter that have a chord length of about 5 feet, though it operates at only 112 RPM and has a much higher tip velocity of about 675 feet per second. Similarly, the flapping (or coning) angle will vary largely from the balance of moments about the flapping hinge as a result of centrifugal and aerodynamic forces, as well as the type of hub that is used. The typical coning angle is very small, only a few degrees in most cases, though for large diameter rotors that may mean that blade tips are deflected a great deal from an undeformed position. In addition, if the blades are designed to have any flexibility in bending then there will be an elastic component to the flap-wise deflection curve. The challenge is in finding a method to measure blade deformation across this wide range of geometric scales and operating conditions.

Currently, measurements of strain on helicopter rotor blades is limited by localized strain gages mounted at discrete span-wise locations as shown in figure 1.1. The lack of deformation data over the entire blade span has led to the use of assumed deformations and interpolated data for the validation of aerodynamic analyses ${ }^{13,14}$. In addition to those already listed, the most important benefit to using full-field optical measurement techniques, such as DIC, is the fact that single-point strain gauges are incapable of showing strain gradients and could potentially miss high strain hot spots. DIC is also more robust than conventional strain gages which often fail during testing. 


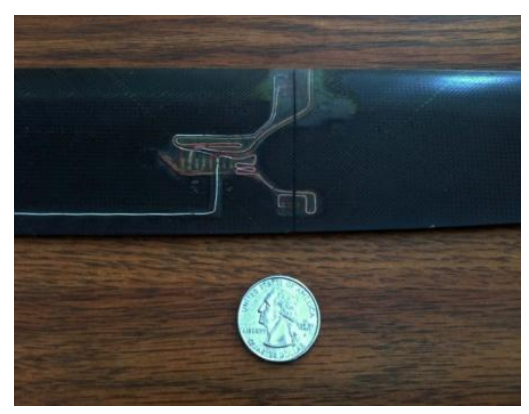

Figure 1.1: Strain gage mounted on a 0.15 scale Bell 412 helicopter blade

There has been some limited investigation of optical measurement techniques for rotor blade deformation. An optical method known as Projection Moiré Interferometry (PMI) has been used to obtain 3D deformation measurements of rotor blades ${ }^{15}$ and has demonstrated superior attributes to conventional approaches. A similar experiment has been performed recently using photogrammetry to measure the deformation of full-scale UH-60 helicopter blades ${ }^{16}$ at various advance ratios, thrust coefficients, and drive shaft angles. The experiment used 2" reflective circular targets attached at specific locations on the blade and produced results that closely matched the design parameters. While this experiment was comprehensive in its testing of a single rotor and demonstrated the accuracy of photogrammetry applied to rotating helicopter blades, it is limited in its resolution and range of applicable scale. DIC has also been used to measure membrane deformation of rapidly actuated flapping wing micro air vehicles (MAVs) ${ }^{17}$.

\section{Digital Image Correlation}

While PMI has almost no sensitivity for in-plane deformation, and moderate sensitivity for out-of plane deformation, DIC has a 3-D sensitivity that can be 1/30,000 of the field of view ${ }^{1}$. Additionally DIC is not affected by rigid body motions and can measure large deformations as long as the test object remains within focus of the cameras. 
Two-dimensional DIC is commonly used in materials testing to obtain in-plane deformation measurements. Previous studies have provided a solid foundation for the advancement of DIC technology since the early 1980's. The technology has been extensively explored in measuring deformations in material sciences and biomedical engineering for a variety of systems such as artificial muscles and ligaments ${ }^{1}$, high speed fracture flywheel ${ }^{7}$ specimens, tensile test of a knee tendon ${ }^{8}$, dynamic tissue deformation measurements of a frog heart ${ }^{9}$, high-speed ballistic impact ${ }^{10}$, and others. Kahn-Jetter and $\mathrm{Chu}^{6}$ presented an overview of DIC theory with experimental verification on a cantilever beam, as well as a brief discussion on methods of random speckle pattern and photogrammetric principles. They concluded that the technique is viable for determining $3 \mathrm{D}$ displacements, the effect of camera tilt is negligible and increasing the camera magnification improves the results. McNeill et al. ${ }^{11}$ discussed the differences between several DIC approaches and calibration methods. Several studies have addressed the advantages of using DIC over single-point conventional strain gages. Siebert et al. ${ }^{12}$ measured the strain at three points on a vibrating cantilever beam using conventional strain gages and compared the results to the strain measured using DIC. They found that the results matched very closely with the exception that the DIC had a greater range of possible deviation due to propagated error. They also explored the sources of error and the propagation of errors in the DIC measurements and found that calibration of the imaging system is one of the largest sources of error. It has been shown that in the right conditions the total error can be reduced to within $0.05 \%$. 


\section{Chapter 2: DIC Physical Principles}

This thesis explores the application of stereoscopic DIC to measure deformation on rotor blades under rotation. The technique is validated by measurements from a laser displacement sensor (LDS) on a simplified vibrating cantilever beam experiment. The goal is to offer a 3D, full-field, non-contact alternative for strain measurement of rotor blades that offers superior spatial resolution compared to traditional strain gages, is cost effective, and simple to implement.

\section{Digital Image CoRrelation TeChNiQue}

In the DIC technique, the surface of the model is first prepared with a random dot or "speckle" pattern such that points on the surface are easily distinguished (see Fig. 2.1). Although various methods exist for creating such patterns, the most reliable method is to spray high-contrast paint on the surface ${ }^{6}$. A high resolution camera captures images of the surface before and after deformation. Cross-correlation of these images yields a twodimensional, or in-plane, displacement map of the surface.

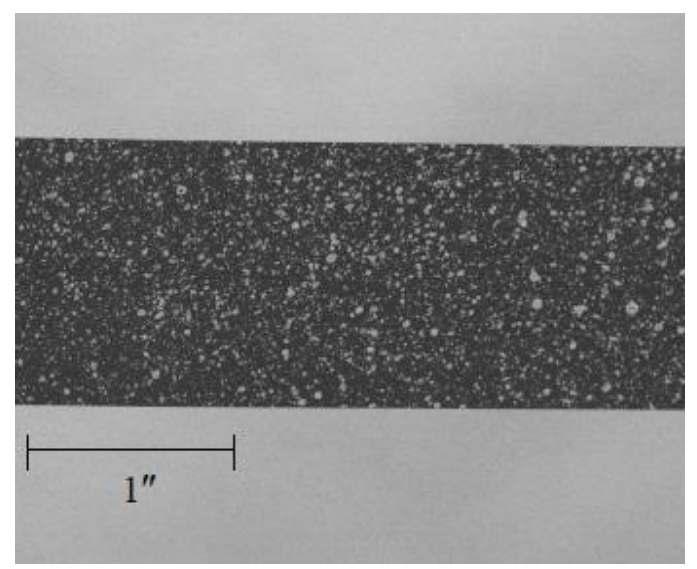

Figure 2.1: Close-up of example speckle pattern 
The application of photogrammetric principles to two-dimensional DIC allows for the determination of three-dimensional contours, non-planar displacements and strain of more complex structures ${ }^{6}$ : this is known as three-dimensional DIC. In this technique, two high resolution cameras are oriented with a line of sight to the test surface, separated by a specific amount such that their respective views overlap (see Fig 2.2). The surface height of a particular point on the article's surface can be determined from the stereoscopic disparity, or parallax between the two camera images. This is defined by the offset of the image between the image-sensing planes of the two cameras ${ }^{6}$, and is calibrated using a target of known geometry. Cross-correlation of images captured by the pair of cameras before and after deformation yields a three-dimensional displacement map of the surface.

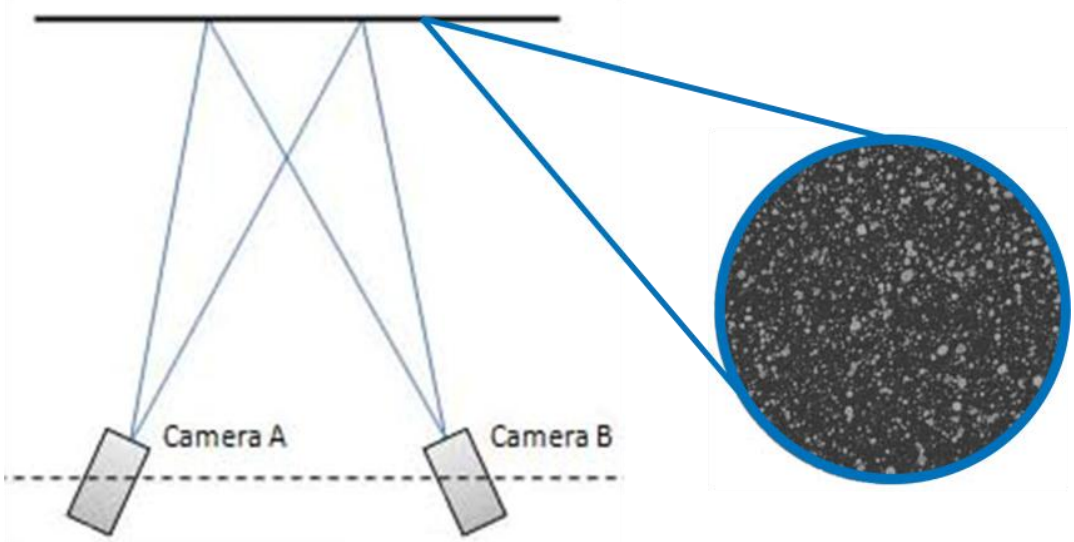

Figure 2.2: Typical stereoscopic DIC setup 


\section{EXPERIMENTAL SETUP}

The steps to setup an experiment using stereoscopic DIC consists of:

1. Hardware Setup

2. Test Article Preparation

3. Dynamic Timing

4. Obtain Proper Lighting and Image Quality

These are described in further detail below.

\section{Hardware Setup}

The cameras are Imager ProX 2M which have 1600x1200 pixel resolution, $29.5 \mathrm{~Hz}$ operation speed, color CCD image sensors, internal camera memory, and 16384 (14-bit) gray levels. The exposure time is adjustable within the range of 500ns to $1000 \mathrm{~s}$ and the cameras are capable of accepting an auxiliary TTL triggering signal. The cameras were equipped with Nikon 50mm AF NIKKOR f/1.8D lenses; these lenses do not have any zoom control and a minimum focal distance of $1.5 \mathrm{ft}(0.45 \mathrm{~m})$, though they have a variable aperture from $\mathrm{f} 1.8$ to $\mathrm{f} 22$. The DIC software used in this thesis is Lavision DaVis 7.2 - StrainMaster 3D ${ }^{18}$.

The rotor blade tests were performed on two separate hover test stands, both of which were designed and built in-house. The first test stand utilizes a two-bladed rigid rotor hub mounted directly to a brushless out-runner DC motor (Hacker A50 16S); this test stand can drive a rotor of up to $2 \mathrm{ft}$ in diameter with a tip speed of 440 feet per second. Three high-speed digital servos operate a swash-plate assembly, allowing for precise control of the rotor collective and cyclic pitch angles. A magnetic $1 /$ rev pickup is used to measure the rotational speed and also provides a trigger for the DIC system or a strobe light. The assembly is mounted to a six component load cell (ATI Mini40E) 
which can measure up to $5 \mathrm{lbs}$ of thrust. Data for this test stand was acquired by a National Instruments CompactDAQ with a custom Labview virtual instrument.

The other test stand, which is used for the larger 39" diameter rotor blades, has a four-bladed aluminum hub assembly with an articulated flap hinge. The hub is mounted to a brushless Hacker A150-8 DC motor, the rotor diameter is limited by test space requirements (approximately $3.5 \mathrm{ft}$ ). The motor is powered by a $10 \mathrm{~kW}$ (max) Lambda TKE ESS 50-200 programmable DC power supply that outputs $200 \mathrm{~A}$ at up to $50 \mathrm{~V}$, and is ultimately torque limited at a maximum of $7.4 \mathrm{ft}-\mathrm{lbs}$ and approximately $133 \mathrm{~Hz}$. The same type of servo/swash-plate assembly as used on the other test stand is used to precisely control the rotor collective and cyclic pitch. A custom fabricated optical 60/rev encoder is fixed to the motor and used to measure the rotational speed and is converted into a $1 /$ rev TTL signal for providing a trigger to the DIC system or a strobe light. There is not a load cell attached to this rotor test stand.

\section{Test Article Preparation}

The high contrast random pattern applied to the test article surface is meant to assist the correlation algorithm with finding patterns in consecutive images. As mentioned previously, the most reliable method to apply a stochastic speckle pattern to the surface is by spraying it with a high-contrast, non-reflective paint. For the experiments presented in this thesis a flat black or white coat was applied to the test article surface followed by a speckle pattern of opposite color; the speckle pattern could be applied either by using a toothbrush dipped in the paint and flicking it over the test article (see Fig. 2.3) or by spraying a light coat from a distance. The former is more appropriate for larger test articles while the latter produced finer speckles better suited to small test articles. It was observed that using black speckles on a white flat coat was 
easier to work with as the test article would have greater average intensity and better contrast with the background.

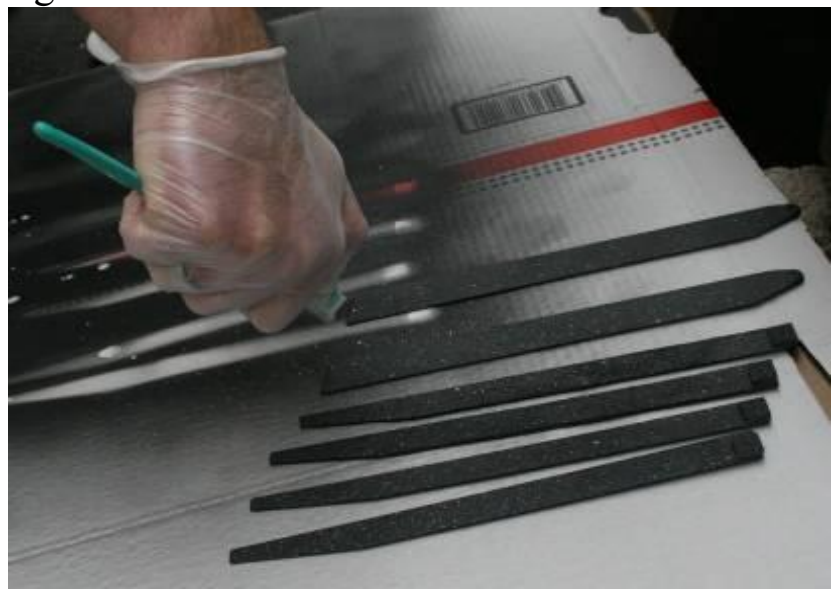

Figure 2.3: Application of a stochastic speckle pattern via the toothbrush method

\section{Dynamic Timing}

The first objective upon setting up each experiment is to ensure that the cameras are synchronized to the motion of the article being tested. This is accomplished by means of a trigger signal generated by the test article. For rotor blade deformation, a magnetic $1 /$ rev pickup is used. Image frames can then be captured at the same point in the cycle of motion. If an image of a fast-moving article lacks definition then the exposure time on the cameras would need to be reduced in order to enhance pattern distinction; however, this often means that more light is required.

\section{Lighting and Image Quality}

In addition to a high contrast random pattern on the article surface, one of the most important characteristics of quality images for DIC is proper lighting. Any digital camera is capable of recording a certain range of light intensity and each pixel in the image will have an intensity rating measured in counts. The experiments presented in this thesis used 14 bit CCD cameras which are capable of intensity in the range 0 to 
16383 counts. An ideal image will have high enough lighting and contrast that the average counts on the article surface will be high while the average counts in the background will be low. If the maximum count number on the article surface is low then it is necessary to provide more light without saturation of the camera. For the experiments presented in this paper all illumination was provided by either a halogen lamp or a strobe; however, experiments involving faster moving objects may require even higher intensity short-duration pulses (i.e. a laser). With proper lighting it should be possible to achieve an average count value of more than 80 counts which is the minimum value to obtain useful correlation results ${ }^{18}$. Some other issues to be aware of are reflections and dust particles on the camera chip, these create bright and dark spots respectively that stay relatively fixed while the test article moves and leads to problems separating the motion of the test article and the fixed pattern. Both of these issues are easily handled through restricting reflective surfaces from being in the camera's field of view and by proper camera care and maintenance respectively.

\section{EXPERIMENT PROCEDURE}

The procedure to measure deformation using DIC consists of the following steps:

1. DIC Calibration

2. Image Acquisition

3. Mask definition

4. Surface height determination

5. Deformation calculation

These are described in detail in the following sections. 


\section{DIC Calibration}

Calibration defines an internal representation for the position and orientation of the cameras relative to the test article. This process determines a scaling factor which ultimately allows for results to be presented in terms of the article's true dimensions. Stereo measurements require a calibration in multiple planes; so a polynomial calibration which uses either a 3D calibration plate or multiple coplanar views of a $2 \mathrm{D}$ plate is required. For our experiments a 3D calibration plate was used. For each view of the calibration target, each camera must obtain images simultaneously. Although one view is typically sufficient to correct for all distortions, providing several views can make the result more accurate ${ }^{18}$. The calibration process implies that the test article is located at the $0 \mathrm{~mm}$ position. Once all of the camera views have been obtained, all of the distinct marks on the calibration plate must be identified. The Lavision software then calculates the mapping function from the known position of the marks on the calibration plate to the cameras. An average deviation of 1 pixel or lower between the calculated mark positions to the ideal grid is considered a good calibration ${ }^{18}$; the software will calculate this deviation automatically, though it is up to the user to confirm the quality of the calibration.

\section{Image Acquisition}

A dataset needs to start with an image of the undeformed test article and follow with any number of images of the deformed test article at a fixed test condition; for example, the rotor experiments typically start with an image of the rotor blade at low speed and zero collective, and are followed by 50-100 images at the flight condition desired for the test. To obtain this, the Lavision DIC software enables the user to put an image acquisition task inside a loop; the image acquisition must be set to obtain a single image, while the loop will repeat for a specified number of times (50-100 in the previous 
example). The images will then be collated into a video file with the image of the undeformed test article as the first image in the video.

\section{Mask Definition}

To perform the image processing it is often necessary to define a mask that will exclude pixels that are not a part of the test article; the surface height will be calculated only for parts of the test article seen by both cameras and defined valid by the mask. The mask can be geometrically fixed for all images, a moving mask fixed to structures, or algorithmically determined based on intensity counts for all images in the set.

\section{- Geometrically fixed mask}

- The simplest option in which a geometric shape is manually drawn around the test article and excludes all pixels that are outside of that shape from calculations.

If the test article moves outside of that shape then data will be lost.

\section{- Moving mask}

○ Determines how the test article has deformed based on the location of identifying marks on the article's surface and adjust the shape of the mask accordingly before calculating the new surface height.

- When deformations are large, this approach can lead to significant loss of data.

\section{- Algorithmic mask}

- Excludes pixels that are not within a specified intensity count range; ideally the test article should have much greater intensity counts than the background, so using an algorithmic mask will automatically mask out all pixels that are not a part of the article's surface regardless of its shape. 
- Can be recreated for every image in the set based on the same options which eliminates the possibility of the mask deforming.

- Can be combined with a geometric mask as well.

In figure 2.4 there is an image with a noisy background in which the DIC algorithm may not be able to differentiate between the background (brown and black speckle) and the object surface (white and black speckle); on the right a mask has been applied which sets all background intensities to zero. In this case, a geometric mask would be most appropriate.
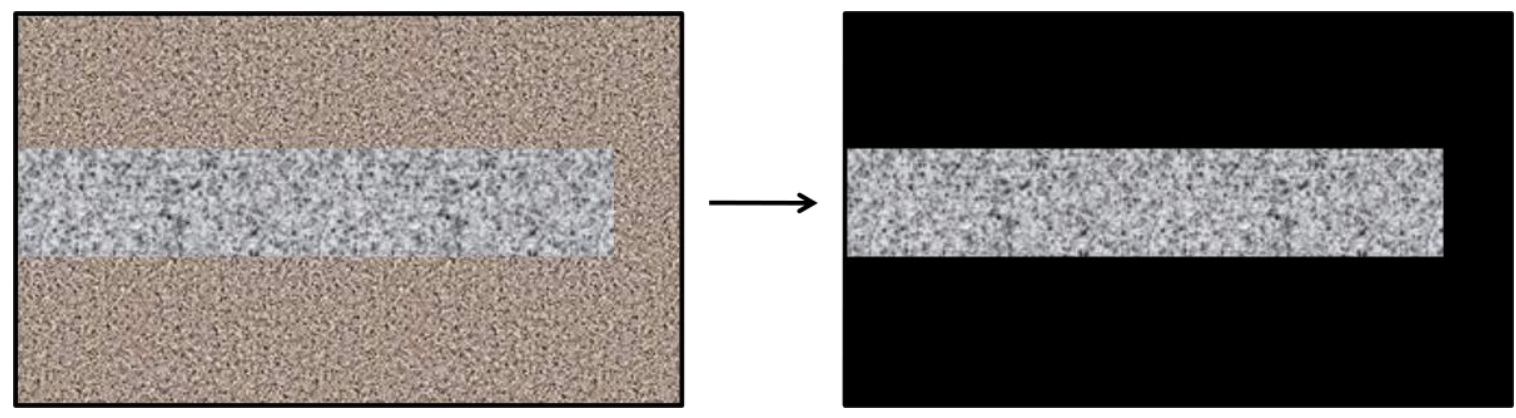

Figure 2.4: Example of image masking; provides a clear boundary between background noise and test article speckle pattern

\section{Surface Height Calculation}

Given a pair of camera images, one for each camera, the surface height can be calculated from the $\mathrm{x}$ and $\mathrm{y}$ coordinates of groups of pixels in both images and the mapping functions that were defined automatically during the camera calibration process. A correlation process identifies the same random pattern in both images and determines the location of interrogation points. The spatial resolution of the technique is determined by the size of the interrogation window which specifies the distance between neighboring points where deformation is calculated. The corresponding points (x1, y1) and (x2, y2) 
in the images from cameras 1 and 2 respectively are then passed through the mapping functions such that the elevation of that group of pixels can be determined, as illustrated in figure 2.5 .

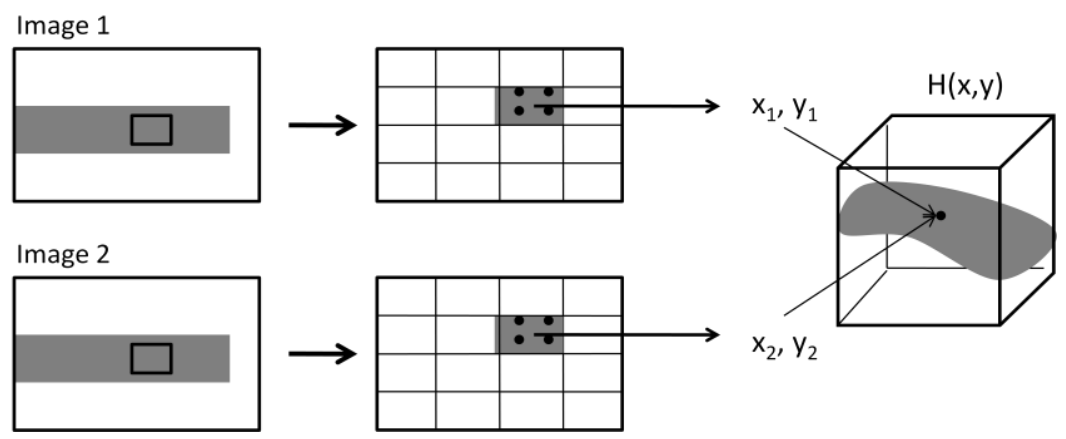

Figure 2.5: Surface Height Calculation

This is sufficient if the surface is smooth and the height is nearly constant; however, as this usually is not the case, an iterative process becomes necessary. That is, the surface height obtained in the aforementioned way becomes an initial guess. The camera images are recalculated under the assumption that the initial guess is correct and sent through a process which calculates residual pixel shifts between the new images. This residual pixel shift is translated into height correction vectors which alter the shape of the initial surface which is then used as a new initial guess for the next iteration. This process is repeated multiple times, reducing the residual pixel shift each iteration in order to further enhance the accuracy of the surface height calculation ${ }^{18}$.

This process is repeated for every pair of images in a set of recorded images and has to be done very carefully for the first image in the set; however, subsequent images can use the result of the preceding image as the initial guess. This implies that the deformation is small enough that consecutive surfaces do not change substantially. Though it is possible to calculate each surface from generalized settings, this takes much more time to compute. 


\section{Deformation Vector Field Computation}

Once the surface height is calculated for all images in a set, the vector deformation calculation is performed by comparing each surface height to the surface height of the first image in the set. The image is divided into interrogation windows in which a single deformation vector is computed for each window. The $2 \mathrm{D}$ vector shifts $\mathrm{u}$ and $\mathrm{v}$ are found for each camera, which are then passed through the mapping functions along with the surface height distribution to obtain the 3D displacement vector $(\mathrm{u}, \mathrm{v}, \mathrm{w})$ of each point as illustrated in figure 2.6.

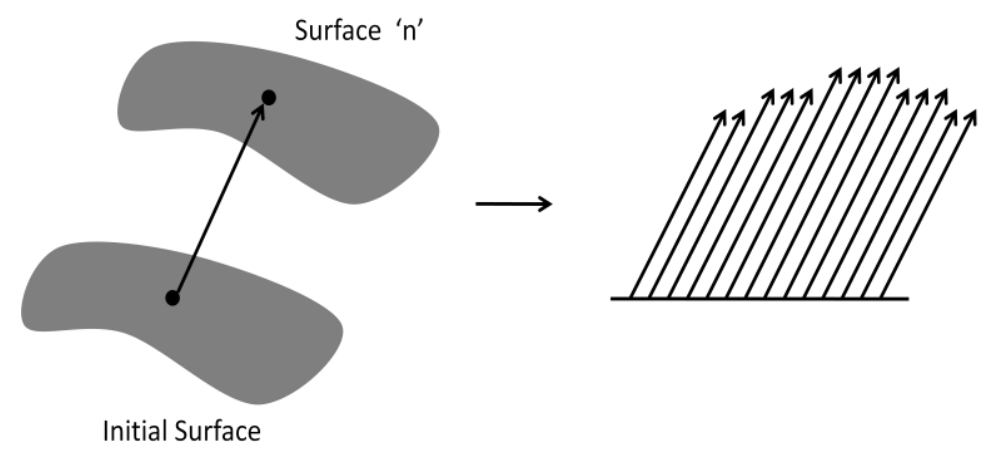

Figure 2.6: Deformation vector field calculation

A variety of postprocessing options are possible in the Lavision software including: eliminating vectors that are not consistent or differ from their neighboring vectors by a specified factor, inserting vectors in empty spots in the vector field, or smoothing. During the smoothing process, errors introduced by outlier vectors are distributed to the 8 neighboring vectors, resulting distributed error is only $1 / 9$ th the original deviation and makes the iteration process more stable ${ }^{18}$. 


\section{ERror ANALYsis of DIC Measurements}

In addition to the small error introduced by smoothing the vector deformation field as mentioned in the previous section, errors are also introduced by other factors in the process. During the surface height calculation an interrogation window was defined which determines the distance between neighboring deformation vectors. The accuracy of the vectors depends on the size of this interrogation window and the amount by which each window overlaps. The distance between two neighboring vectors, or the vector grid, is determined by

$$
g=\frac{\text { interrogation window size }}{100 /(100-\% \text { overlap })}
$$

For example, all experiments in this thesis use an interrogation window size of $32 \times 32$ pixels with $50 \%$ overlap which will have a vector grid of 16 pixels and a corresponding $3 \mathrm{D}$ vector precision of 0.05 pixels as determined by a Lavision software look-up table. The accuracy depends largely on the interrogation window size, but also on the zoom factor of the cameras, the type of lens used, and the distance to the test $\operatorname{article}^{18}$. Larger vector grids are more accurate; however, they reduce the spatial resolution and may not resolve local discontinuities. So, it is important to choose a window size that offers the best compromise between required spatial resolution and accuracy. 


\section{Chapter 3: Experiments}

Several experiments were conducted involving the stereoscopic DIC technique including a simple vibrating cantilever beam, two different diameter rigid rotor blades (24" and 39"), as well as 18" flexible MAV rotor blades. The cantilever beam served as a foundation so that the technique and the results it produced could be well understood, it also allowed for experimental verification of the DIC results through the use of a laser displacement sensor (LDS).

\section{CANTilever BeaM}

The first phase of the study validated the DIC system on a simple dynamic model; a vibrating aluminum beam. A piezoelectric actuator attached near the base of the beam excited the beam's second natural frequency (at around $36 \mathrm{~Hz}$ ). The DIC was used in conjunction with a Laser Displacement Sensor (LDS) to verify the displacement measurements. A stochastic white speckle pattern (see Fig. 3.1.1 \& 3.1.2) on a black coat was used. The cameras were triggered at the frequency of vibration of the beam such that they synchronized with the vibration of the beam. The entire period of motion was recorded by incorporating a delay between the trigger signal and the image capture; a total of 20 images composed a set. The cameras used have a fixed zoom factor and the field of view could not be adjusted except by moving closer to or further away from the test article. As the entire beam did not fit inside the camera field of view it was necessary to combine multiple data sets in order to get the full length of the beam; this was accomplished by using a particular speckle which is overlapped in two views as a reference point and shifting the data along the span such that the curves overlap at that point. This can be done whenever there are no changes to the DIC calibration; if the DIC calibration is changed it will change the spatial resolution in the horizontal and vertical 
axes and create large discontinuities in the measurements. The main goal of this experiment was to quantify the errors of the system and understand the limitations of the technique with respect to dynamic displacement measurement.

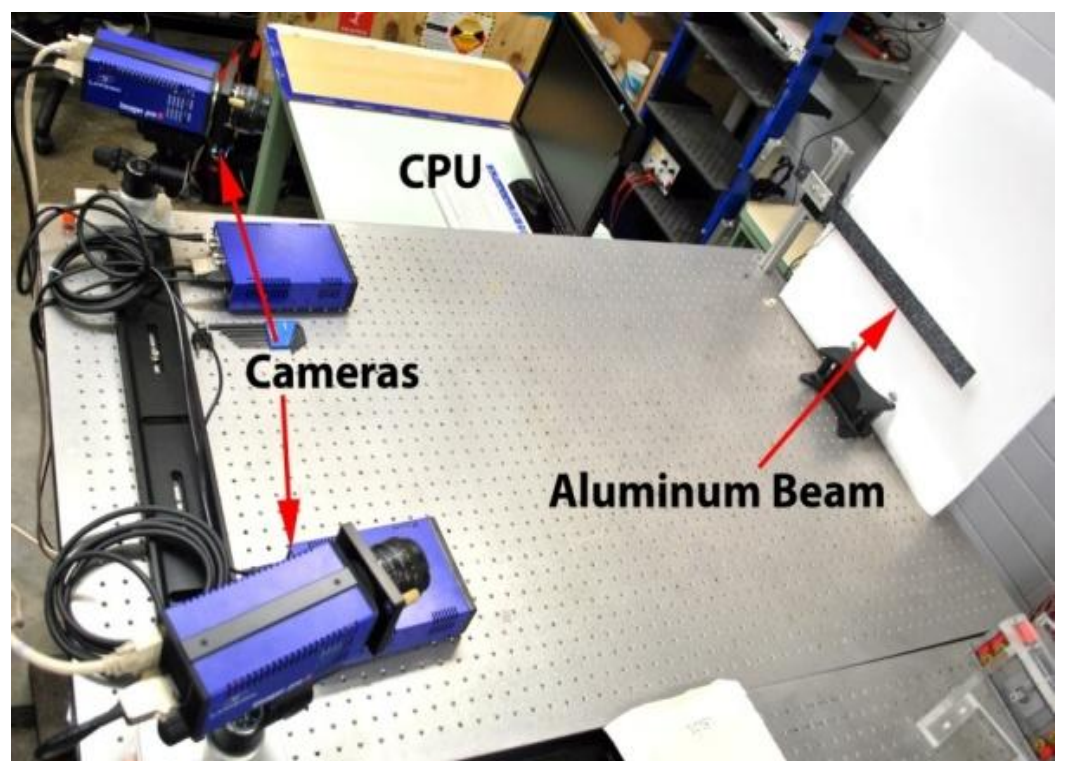

Figure 3.1.1: Experimental setup for measuring the deflection of a vibrating cantilever beam using DIC. 


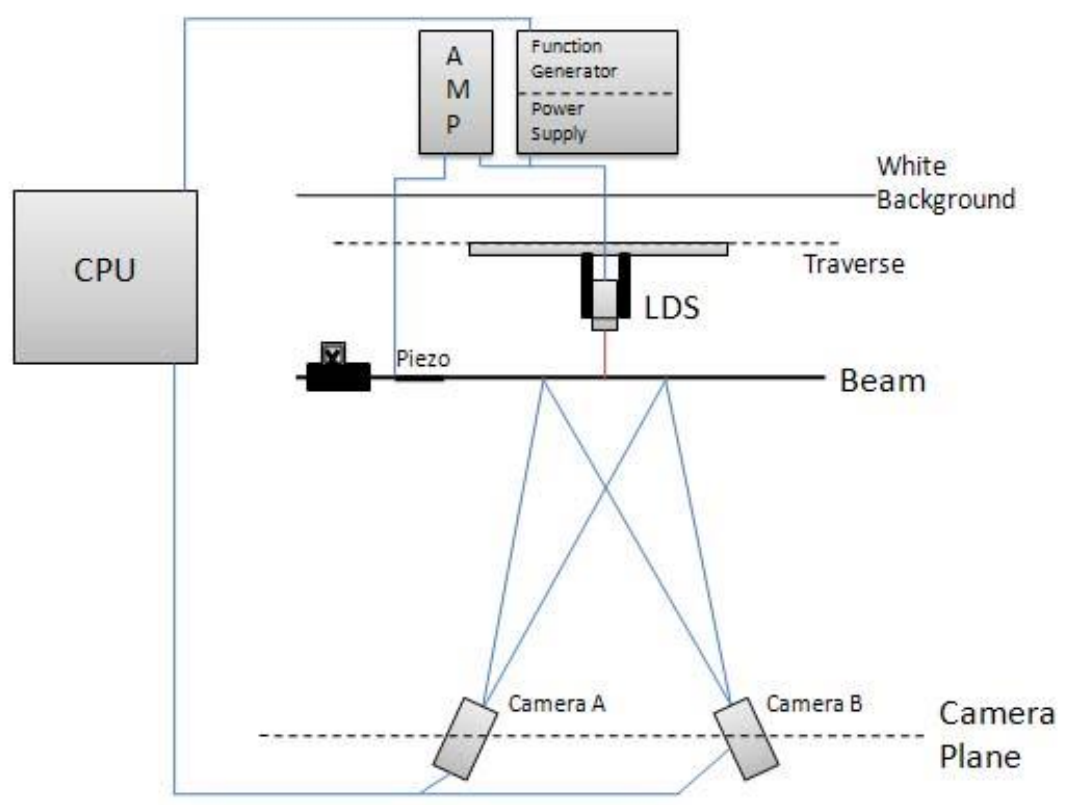

Figure 3.1.2: Schematic for vibrating cantilever beam experiment.

The light source for this experiment was ambient room light as it was sufficient to capture the motion, and since motion was predominantly in the z-axis a moving geometric mask was used. A $32 \times 32$ pixel Interrogation window with $50 \%$ overlap was used in the surface height calculation. As mentioned before, the entire beam could not fit inside the field of view of the cameras and was split into multiple segments. The results in figure 3.1.3 were obtained using two separate segments of DIC data and are compared to the results of the LDS measurements. The error bars in the figure correspond to the accuracy of the LDS; it is of considerable importance that the DIC results are within the accuracy of the LDS. The experiment was repeated with four separate segments of the beam and the bending mode shape was extracted from the data and reconstructed as a continuous curve. The deformed shape of the beam was assumed to take the shape

$$
w(x)=C_{1} \cos (B x)+C_{2} \sin (B x)+C_{3} \cosh (B x)+C_{4} \sinh (B x)+\beta_{0} x
$$


where $B$ is a constant determined by structural properties, $C_{i}$ and $\beta_{0}$ are constants to be solved for based on the available data. The second derivative of this curve can then be combined with Euler-Bernoulli beam theory in order to obtain the strain:

$$
\varepsilon=-z \frac{\partial^{2} w}{\partial x^{2}}
$$

Here $\mathrm{z}$ is the distance to the neutral axis of the beam (or half the thickness). The reconstructed curve in figure 3.1.4 is derived by fitting equation (2) to the data obtained from DIC, and figure 3.1.5 shows the strain as derived by equation (3).

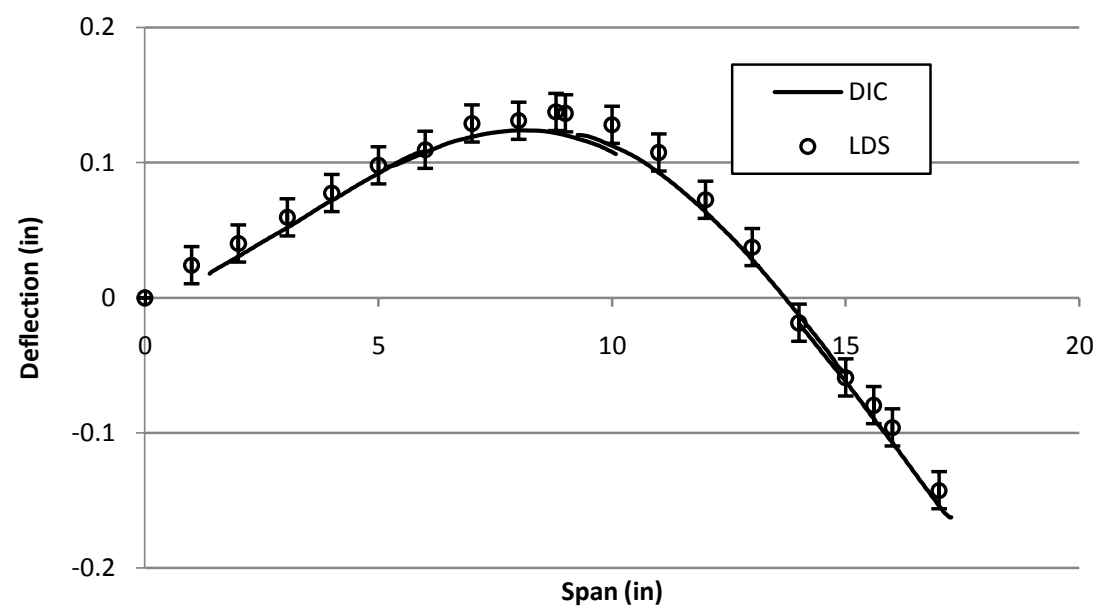

Figure 3.1.3: Cantilever beam deflection measurements from DIC and LDS 


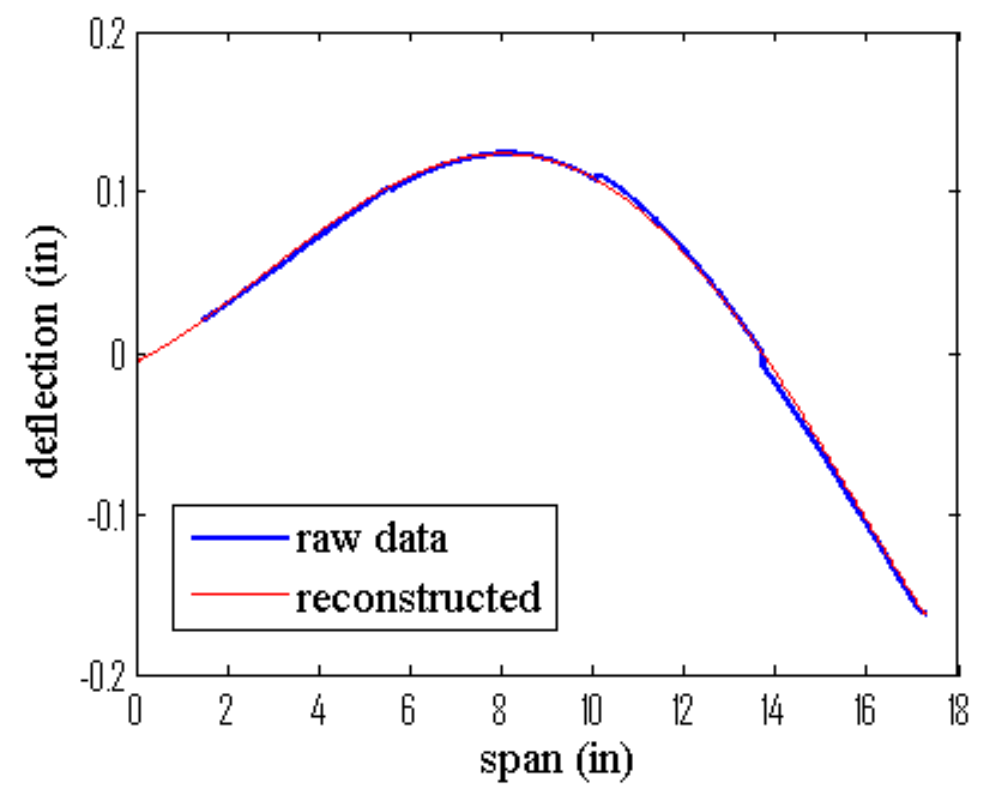

Figure 3.1.4: Deflection vs. span, comparison between raw data and reconstructed curve

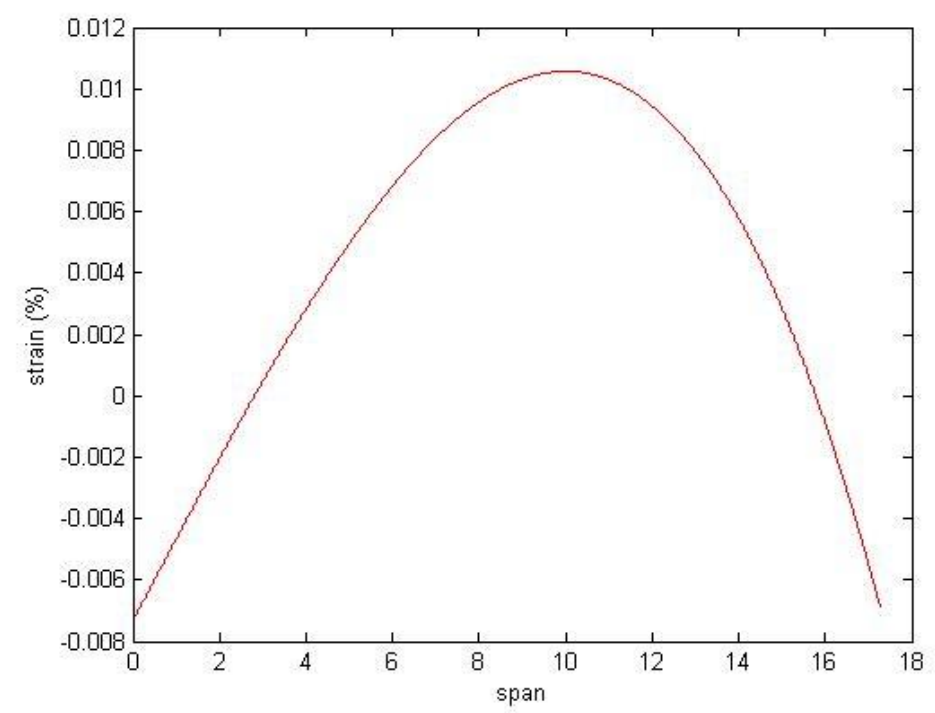

Figure 3.1.5: Strain vs. span for vibrating cantilever beam

It was concluded that the DIC measurements are accurate (within the error bounds of the LDS) and reproducible. Even if the data cannot be obtained as a continuous 
surface the segments can be easily combined and reconstructed into a continuous curve provided that there are no changes to the calibration between the different sets of data. Good correlation between the DIC and theoretical deformation was obtained.

\section{RIGID ROTOR BLADES}

After the DIC measurement methodology was validated on a cantilevered beam, the study progressed to measurements of deflection on helicopter rotor blades. Several different rotors were tested in order to substantiate the technique across a range of scales and testing conditions.

\section{9" Diameter Rotor}

A 39" diameter, four-bladed helicopter rotor using rigid NACA 0014 blades, with 2.05" constant chord length, and an articulated (flap only) hub was tested in hover (see Fig. 3.2.1). A stochastic black speckle pattern on a white background was applied to the rotor blades; it was found that a white background would raise the average intensity counts on the surface and made masking the surface easier for rotating systems in which shorter exposure times are needed. A 1/rev optical trigger on the rotating frame was used to synchronize the rotor with the DIC system. A strobe light was used for illuminating the blade at an appropriate azimuth position. The full-field deformation was measured at four rotational speeds $(400,900,1200$ and $1500 \mathrm{rpm}), 50$ images per set, and two collective pitch angles $\left(3.5^{\circ}\right.$, and $\left.8^{\circ}\right)$. The collective angles were determined by mounting a laser on the rotor and measuring the deflection of the laser on a distant wall for a particular collective input. Deformations were calculated from a $0^{\circ}$ collective baseline at $400 \mathrm{rpm}$ such that there is enough centrifugal force to keep the blades spinning in a horizontal plane (no coning angle), while the force is simultaneously low enough that the induced strain should be negligible at this condition. 


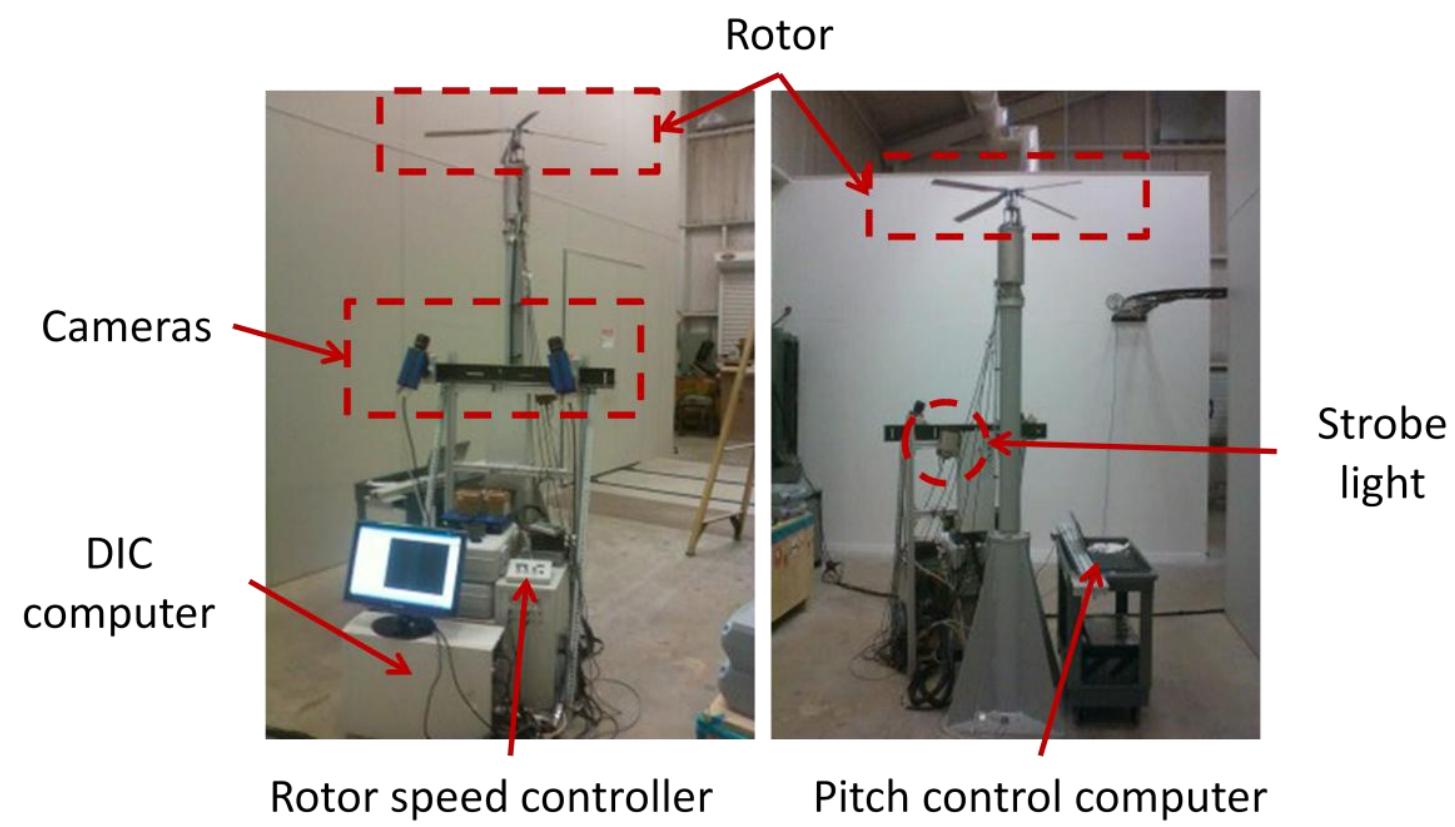

Figure 3.2.1: Experimental setup on the measurement of deformation on 39" diameter rigid rotor blades using DIC

\section{4" Diameter Rotor}

A 24" diameter, two-bladed helicopter rotor using rigid NACA 0014 blades, with 0.95" constant chord length, and a rigid hub was tested in hover (see Fig. 3.3.1 \& 3.3.2). A stochastic, black on white, speckle pattern was applied to the rotor blades and a 1/rev trigger on the rotating frame was used to synchronize the rotor with the DIC system. A 1000W halogen lamp was used to illuminate the blades continuously and phase delays were introduced to capture images at the appropriate azimuth angles. The motor and rotor assembly were mounted directly on a six-component load cell (ATI Mini40E) which was used to obtain force and torque measurements at each test condition. Fullfield deformation was calculated at two rotational speeds (1500 and $1800 \mathrm{rpm}), 100$ images per set, and five collective pitch angles $\left(3.6^{\circ}, 6.1^{\circ}, 8.5^{\circ}, 12.3^{\circ}\right.$, and $\left.14.8^{\circ}\right)$ as measured by the method described for the $39 "$ rotor and verified by an inclinometer. 
Deformations were calculated from a $0^{\circ}$ collective baseline for each rotor speed since the primary interest is flap-wise deformation and pitch measurement. Blade Element Momentum Theorem (BEMT) was applied to the DIC results in order to make a comparison to load cell measurements.

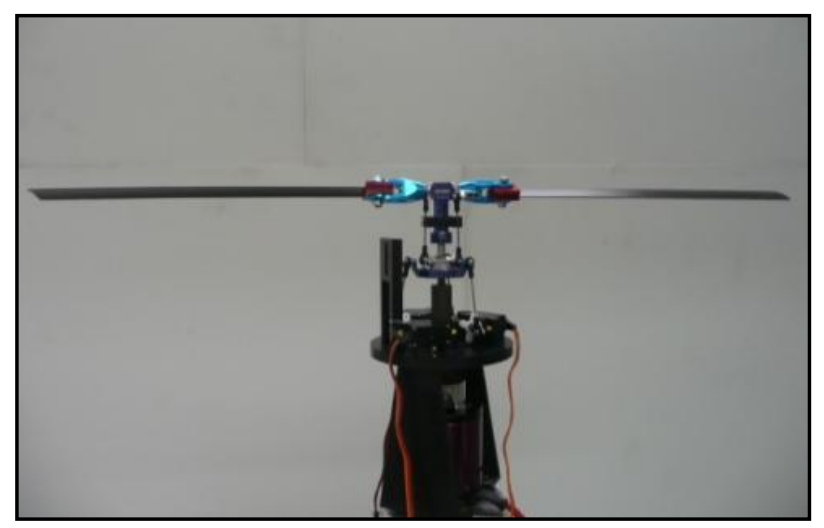

Figure 3.3.1: 24 " rigid rotor blades on the hover test stand

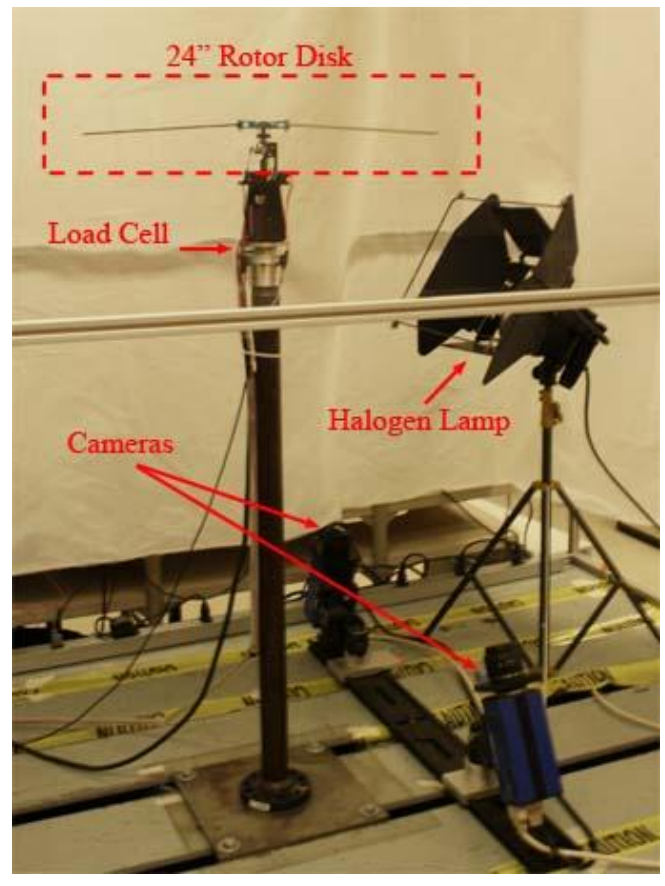

Figure 3.3.2: Experimental setup on the measurement of deformation on 24" diameter rigid rotor blades using DIC 


\section{FLEXIBLE ROTOR BLADES}

The same procedure as described for the $24 "$ diameter rigid blades was used to measure the deformation for 3 separate sets of $18^{\prime \prime}$ diameter, $0.945 "$ constant chord length, flexible MAV blades ${ }^{20-22}$. The setup was nearly identical to the 24 " diameter rigid rotor experiment (see fig. 3.3.2); the same test stand and equipment were used, with the only changes being that since the flexible blades were shorter the cameras were moved such that the entire blade could fit inside the field of view. As with the previous experiments, the blades were painted white with a black speckle pattern. The tests were performed at 1500rpm, 100 images per set, and five different collective pitch angles $\left(4.8^{\circ}, 9.8^{\circ}, 14.8^{\circ}, 19.7^{\circ}\right.$, and $\left.25.9^{\circ}\right)$. Deformations were measured relative to a $0^{\circ}$ collective baseline at 1500rpm.

The flexible blades were fabricated using carbon fiber and an elastomeric matrix with a wet layup process. The blades are so flexible that centrifugal stiffening dominates blade dynamics; the blades cannot support their own weight in non-rotating condition. During operation a large nose-down twist is induced in the blades which results in significantly reduced efficiency relative to rigid blades with comparable planform and airfoil section. This drove the flexible blade design toward modifications that would passively tailor the span-wise twist distribution and improved efficiency. For more detailed description of the construction of the flexible blades see Sicard and Sirohi ${ }^{20-22}$. Three types of flexible blades were tested with subtle differences between them. A close up of the flexible blades on the rotor test stand can be seen in figure 3.4.1 and the blades used in this experiment can be seen in figure 3.4.2. Note that it is not feasible to measure the shape of the rotating blade using conventional means such as strain gages; the goal is to use DIC to evaluate the differences in blade shape during rotation. 


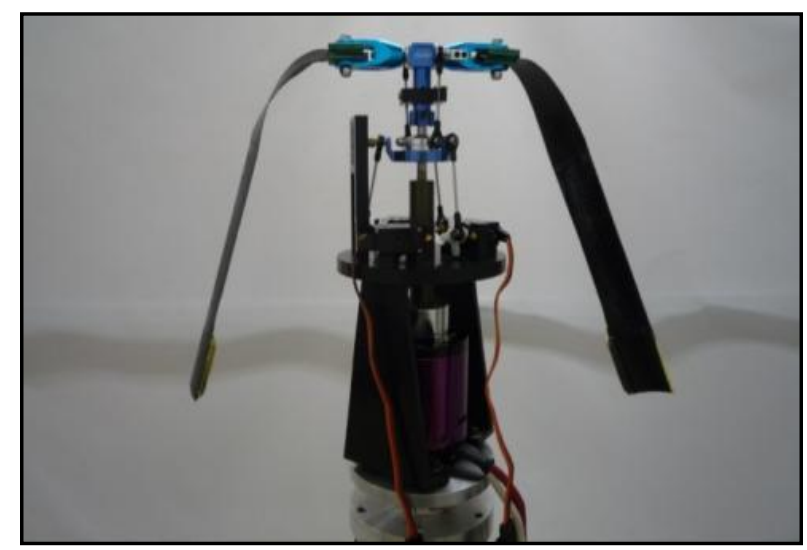

Figure 3.4.1: Flexible rotor blades on the hover test stand

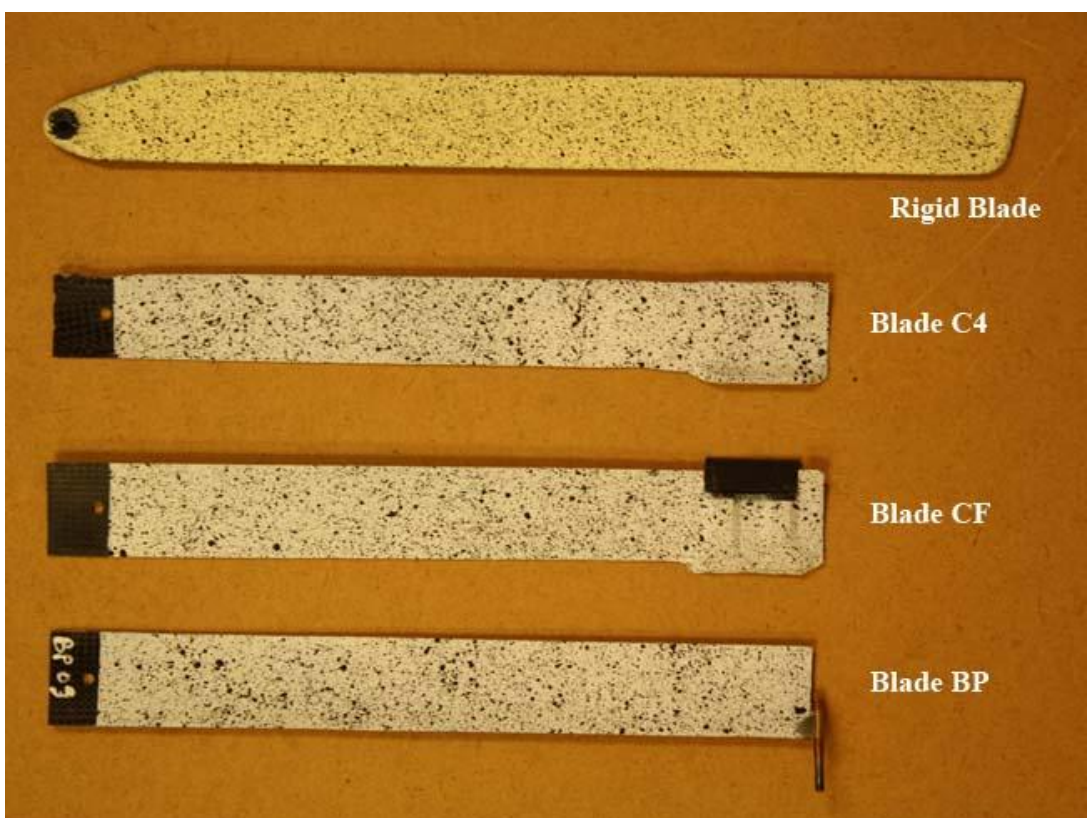

Figure 3.4.2: Comparison between rotor blades used in experiments

The different types of blades tested are described in the following sections. 


\section{Blade C4}

Blade C4 uses an uncoupled composite laminate and represents the baseline. This approach uses two plies of carbon fiber cloth oriented at $\pm 45^{\circ}$ with respect to the blade span impregnated with a flexible epoxy resin and compressed in a mold. One ply of carbon fiber was wrapped around a thin-walled brass tube at the blade tip oriented with its axis parallel to the blade span and a tungsten rod was inserted into the tube in order to increase tip-mass and provide centrifugal stiffening. The geometry of blade $\mathrm{C} 4$ can be seen in figure 3.4.3.

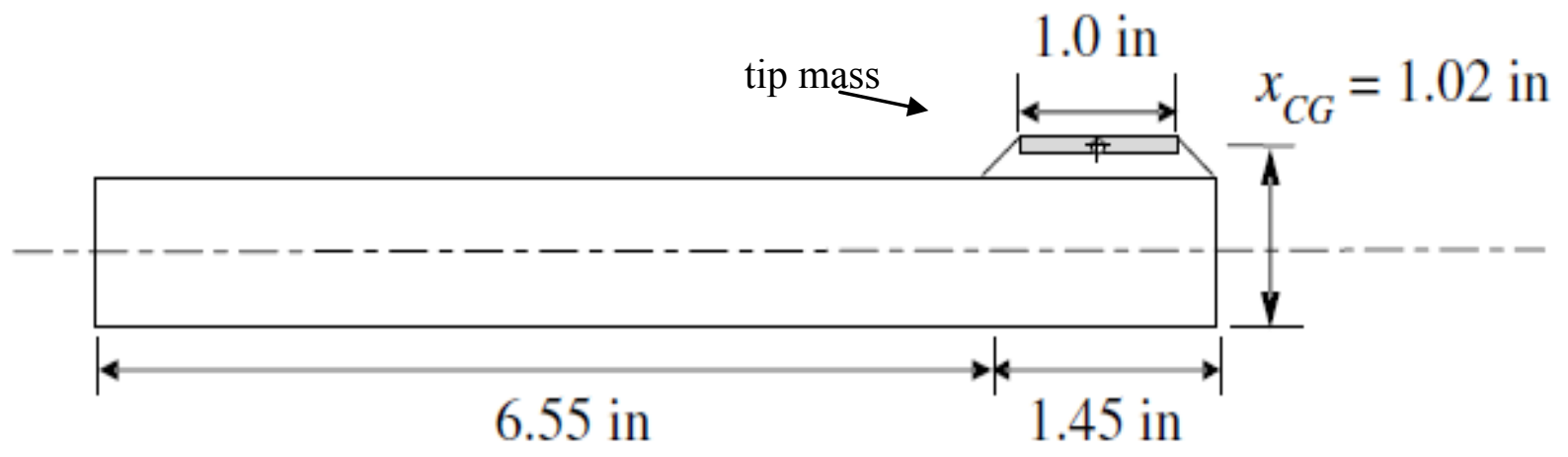

Figure 3.4.3: Physical geometry of blade C4 (adapted from Sicard ${ }^{22}$ )

\section{Blade CF}

Blade CF involves the use of trailing edge flaps in order to produce pitching moment changes ${ }^{21,22}$. This design was based on the previous blade $\mathrm{C} 4$ design with the addendum of a flap mounted at the trailing-edge, aft of the tip body (see fig. 3.4.4). In general, a trailing-edge flap deflected up will produce a nose-up pitching moment about the feathering axis and reduce the unfavorable blade twist. At the blade tip a Kevlar ${ }^{\mathcal{O}}$ sheet encased the tip mass as well as a polymer foam core which the flap could be connected. The flap itself was constructed of 6 layers of 0/90 carbon/epoxy lamina and 
was rigid compared to the blade. Two spring steel supports were inserted at mid-ply of the flap; the angle between the supports and the flap-chord was preset at a desired value and inserted into the blade's polymer foam core ${ }^{22}$.

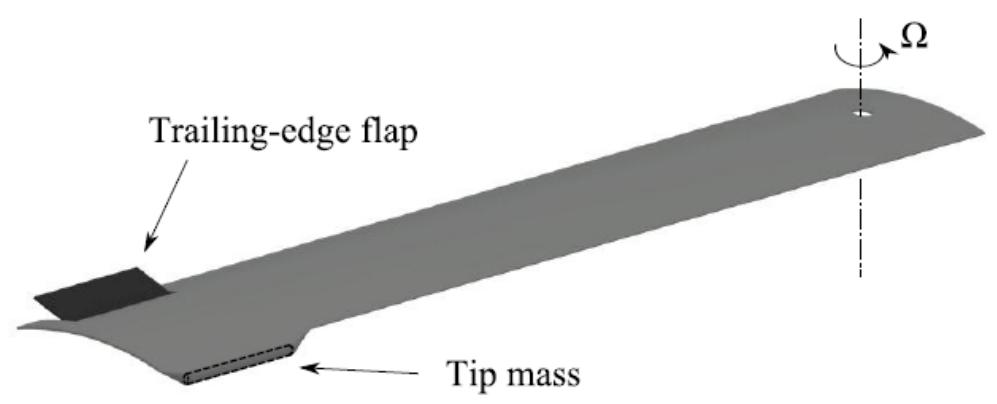

Figure 3.4.4: Flexible blade CF (adapted from Sicard $^{22}$ )

\section{Blade BP}

Blade BP introduces an index angle between the chord of the blade and the principle axis of the tip body in order to make use of a restoring propeller moment ${ }^{21,22}$. In this design the tip mass is secured perpendicular to the span-wise coordinate and at a predefined index angle with respect to the blade chord. The expectation is that while in rotation the propeller moment acting on the tip mass will align its longitudinal axis with the plane of rotation and result in an untwisted blade. The tip mass was soldered to the blade tip such that it made a $22^{\circ}$ angle with the blade chord ${ }^{22}$ as shown in figure 3.4.5.
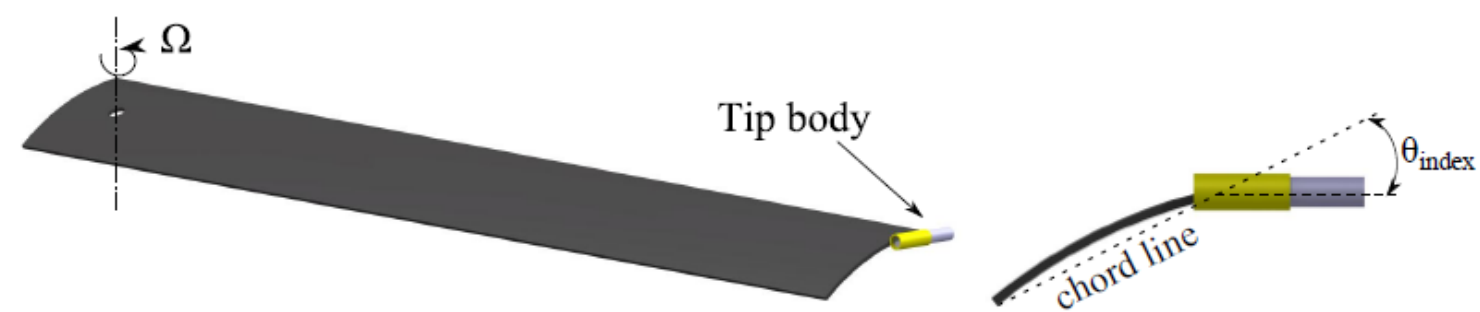

Figure 3.4.5: Flexible blade BP (adapted from Sicard $^{22}$ ) 


\section{Chapter 4: Performance Analysis using Blade Element Momentum Theory}

The blade element momentum theory (BEMT) is a method for calculating the thrust produced and power required by a hovering rotor using principles from momentum theory and blade element approaches. In momentum theory, the rotor can be idealized as an infinitesimally thin actuator disk over which there exists a pressure differential ${ }^{23}$. Conservation of momentum provides a relation between the rotor thrust, $\mathrm{T}$, and the net time rate of change of fluid momentum out of the control volume such that

$$
T=2 \rho A v_{i}^{2}=2 \dot{m} v_{i},
$$

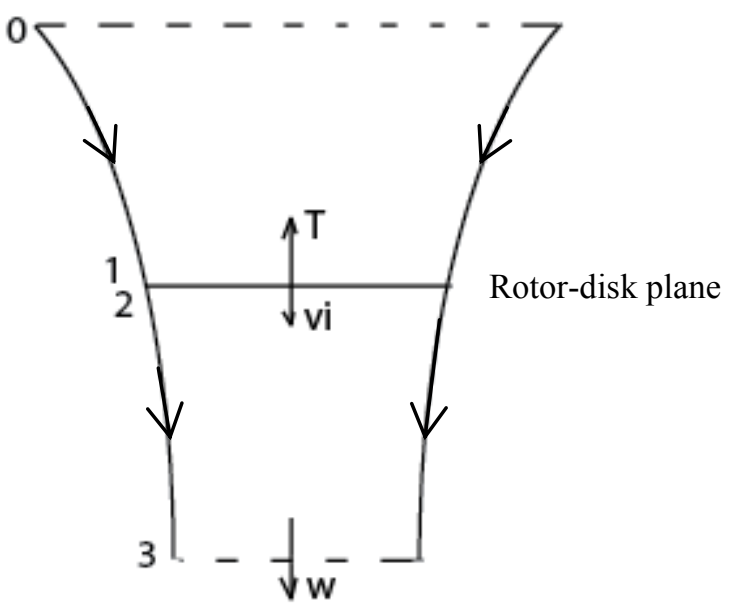

Figure 4.1: Simplified flow model of momentum theory analysis of a rotor in hover

where $v_{i}$ is the induced fluid velocity through the rotor plane and the reference area $\mathrm{A}$ is the rotor disk area. The rotor thrust and ideal power coefficients are then defined as

$$
\begin{gathered}
C_{T}=\frac{T}{\rho A \Omega^{2} R^{2}} \\
C_{P}=\frac{T v_{i}}{\rho A \Omega^{3} R^{3}}=\frac{C_{T}^{3 / 2}}{\sqrt{2}} .
\end{gathered}
$$

Approximating the actual power required to hover requires knowledge of the sectional profile drag coefficient, $\mathrm{C}_{\mathrm{d} 0}$, an induced power correction factor, $\kappa$ (typically 1.15 ), and 
the rotor solidity, $\sigma$, which is a ratio of the rotor blade area to the rotor disk area. The figure of merit, FM, for a particular rotor operating under specific conditions is the ratio between ideal power required to hover and actual power required to hover.

$$
\begin{gathered}
\sigma=\frac{N_{b} c R}{A}=\frac{N_{b} c}{\pi R} \\
F M=\frac{P_{\text {ideal }}}{P_{\text {actual }}}=\frac{\frac{C_{T}^{3 / 2}}{\sqrt{2}}}{\frac{\kappa C_{T}^{3 / 2}}{\sqrt{2}}+\frac{\sigma C_{d 0}}{8}} .
\end{gathered}
$$

The blade element theory (BET) assumes that each blade section acts as a $2 \mathrm{D}$ airfoil to produce aerodynamic forces and moments ${ }^{23}$ as shown in figure 4.2. By integrating the sectional air-loads at each blade element over the length of the blade and averaging the result over a rotor revolution, the rotor performance can be obtained. Unlike momentum theory, BET utilizes blade twist, planform distribution, and airfoil shape to evaluate rotor performance.

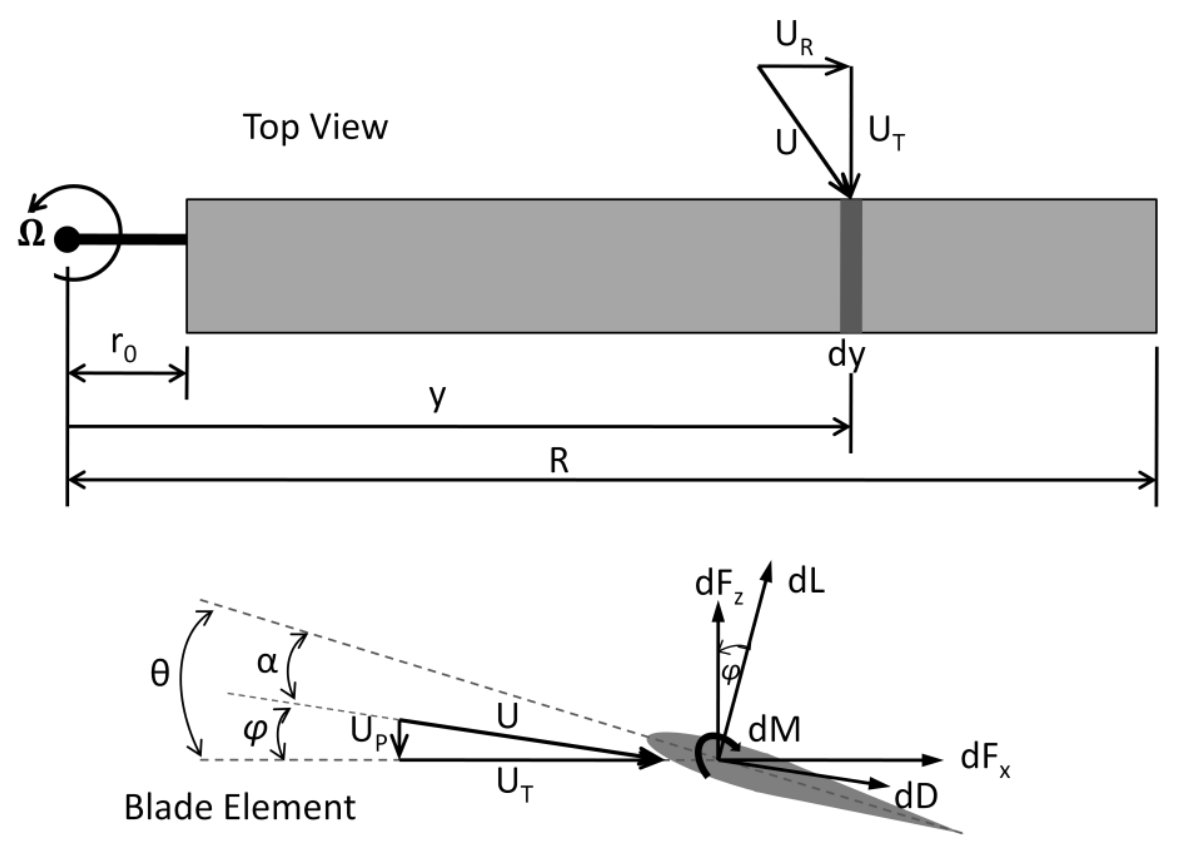

Figure 4.2: Aerodynamic forces and moments acting on a typical blade element; illustration of blade physical characteristics. 
From the diagram, it is apparent that the relative inflow angle, $\varphi$, at the blade element will be dependent on the local flow velocity out-of-plane and in-plane components; $U_{P}$ and $U_{T}$ respectively. For the hover case that is

$$
\begin{gathered}
U_{P}=v_{i} \\
U_{T}=\Omega y \\
\varphi=\tan ^{-1}\left(\frac{U_{P}}{U_{T}}\right) \approx \frac{U_{P}}{U_{T}} \text { for small angles }
\end{gathered}
$$

and thus for a blade element pitch angle, $\theta$, the effective angle of attack is

$$
\alpha=\theta-\varphi=\theta-\frac{U_{P}}{U_{T}} .
$$

The incremental lift and drag per unit span are then

$$
d L=\frac{1}{2} \rho U^{2} c C_{l \alpha} \alpha d y \quad \text { and } \quad d D=\frac{1}{2} \rho U^{2} c C_{d \alpha} \alpha d y .
$$

Resolving these incremental forces into components perpendicular and parallel to the rotor disk plane gives the relations

$$
d F_{z}=d L \cos \varphi-d D \sin \varphi \quad \text { and } \quad d F_{x}=d L \sin \varphi+d D \cos \varphi .
$$

Then for a rotor with $\mathrm{N}_{\mathrm{b}}$ blades, the rotor thrust, torque, and power are

$$
T=N_{b} F_{z}, \quad d Q=N_{b} y d F_{x}, \quad \text { and } \quad d P=N_{b} \Omega y d F_{x} .
$$

Similarly the increment in thrust coefficient is

$$
d C_{T}=\frac{N_{b} d L}{\rho A(\Omega R)^{3}} \rightarrow \frac{1}{2} \sigma C_{l} r^{2} d r
$$

where $r$ is a non-dimensional quantity defined by dividing $y$ by the length $R$, that is to say $r=y / R$.

In the BEMT method the conservation laws are considered to act on an annulus of the rotor disk a distance $y$ from the rotational axis with width $\mathrm{dy}^{23}$. The area $d \mathrm{~A}$ of this annulus is then $2 \pi y d y$ and the incremental thrust $d \mathrm{~T}$ can be calculated from the application of momentum theory and Prandtl's circulation-loss function. From momentum theory recall $T=2 \dot{m} v_{i}$ (equation 4). The mass flow rate through an annulus of the rotor disk is then 


$$
d \dot{m}=\rho d x v_{i}=2 \pi \rho v_{i} y d y .
$$

Then applying (17) to (4) gives the relation for incremental thrust on the annulus as

$$
d T=4 \pi \rho v_{i}^{2} y d y
$$

and the incremental thrust coefficient is

$$
d C_{T}=\frac{d T}{\rho A(\Omega R)^{2}}=\frac{4 \pi \rho v_{i}^{2} y d y}{\rho\left(\pi R^{2}\right)(\Omega R)^{2}}=\frac{4 v_{i}^{2} y d y}{\Omega^{2} R^{4}} .
$$

It is often convenient to work in non-dimensional quantities, so we introduce the inflow ratio from BET that can be expressed as

$$
\lambda=\frac{v_{i}}{\Omega R}=\varphi r
$$

which allows (19) to be simplified to

$$
d C_{T}=4 \frac{v_{i}}{\Omega R}\left(\frac{v_{i}}{\Omega R}\right)\left(\frac{y}{R}\right) d\left(\frac{y}{R}\right)=4 \lambda \lambda_{i} r d r .
$$

Similarly, the induced power consumed by the annulus is

$$
d C_{P}=\lambda d C_{T}=4 \lambda^{2} \lambda_{i} r d r
$$

Then by equating the incremental thrust coefficients from blade element and momentum theories we get the relation

$$
\begin{gathered}
\frac{1}{2} \sigma C_{l} r^{2} d r=4 \lambda \lambda_{i} r d r \\
\frac{\sigma C_{l \alpha}}{2}\left(\theta r^{2}-\lambda r\right)=4 \lambda^{2} r \\
\lambda^{2}+\left(\frac{\sigma C_{l \alpha}}{8}\right) \lambda-\frac{\sigma C_{l \alpha}}{8} \theta r=0 .
\end{gathered}
$$

The solution to the quadratic equation in $\lambda$ provides the solution

$$
\lambda(r)=\frac{\sigma C_{l \alpha}}{16}\left(\sqrt{1+\frac{32}{\sigma C_{l \alpha}} \theta r}-1\right) .
$$

In the DIC experiments a lookup table is used to find $C_{1}$ and $C_{d}$ for a given section effective angle of attack and it is simpler to write (24) as

$$
\lambda(r)=\frac{\sigma C_{l}}{16 \alpha}\left(\sqrt{1+\frac{32 \alpha}{\sigma C_{l}} \theta r}-1\right),
$$


which is a radial inflow equation that satisfies both blade element and momentum theories $^{23}$, and can be solved using a known radial pitch distribution which is useful when applied to DIC results.

The radial inflow equation in hover can be improved by incorporating loss of lift near blade tips caused by a finite number of blades ${ }^{23}$. Prandtl posed a revised solution expressed in terms of a correction factor, $\mathrm{F}$, where

$$
F=\left(\frac{2}{\pi}\right) \cos ^{-1}\left(e^{-\frac{N_{b}}{2}\left(\frac{1-r}{r \varphi}\right)}\right)
$$

and the radial inflow equation in hover becomes

$$
\lambda(r)=\frac{\sigma C_{l}}{16 \alpha F}\left(\sqrt{1+\frac{32 \alpha F}{\sigma C_{l}} \theta r}-1\right) .
$$

A problem arises in that the inflow velocity must be known in order to calculate thrust in this way; however, the thrust is required to find the inflow velocity, so it is necessary to have an iterative algorithm which takes an initial guess for the thrust and compares the newly calculated thrust to the thrust from the previous iteration. If a convergence condition has not been met, then the inflow velocity will be adjusted such that it is between the old and new inflow velocities. Utilizing BEMT principles along with given operating conditions, a bending mode distribution, and radial pitch distribution as produced by DIC results it is possible to calculate the expected thrust (equation 21), power consumption (equation 22), and figure of merit (equation 8). A flow chart depicting the BEMT processing on DIC results is shown on the next page, figure 4.3. 


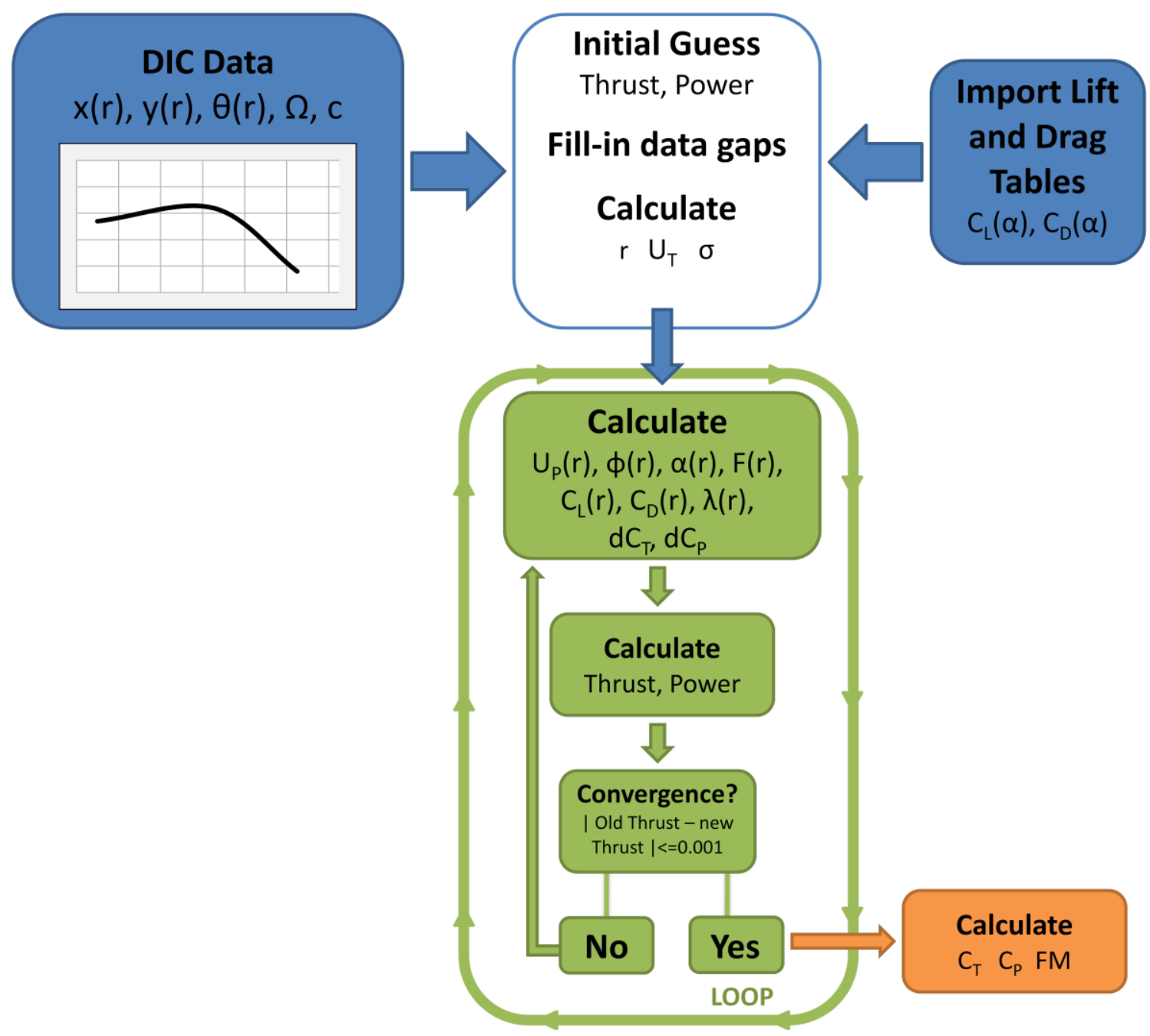

Figure 4.3: Flow chart illustrating BEMT processing of DIC data 


\section{Chapter 5: Results \& Discussion}

The results of the previously discussed experiments are shown in the following sections. From the rigid rotor blades it was observed that DIC results could be obtained for different geometric scales and the outcome agreed with expectations, though pitch seemed to be slightly under-calculated, particularly at higher collective pitch. By applying BEMT to the rigid blade DIC results it was possible to obtain approximations for the thrust, power, and figure of merit for the rotor; all of which were in relative agreement with load cell measurements. The use of a strobe light for illumination was observed to produce more consistent, repeatable results than with the $1000 \mathrm{~W}$ halogen lamp.

The flexible blade results show much more deformation, as expected, and results help to validate the design considerations for the blades. Twist, in particular, is much more noticeable. The fact that these measurements would not have been possible with other conventional methods illustrates the utility of the DIC technique.

Table 5.1: List of experiments and scales

\begin{tabular}{|l|c|}
\multicolumn{1}{|c|}{ Experiment } & Rotor diameter / beam length \\
\hline 1) & (in) \\
\hline 2) Rigrating beam & $17.8^{\prime \prime}$ \\
\hline 3) Large rotor blades & $24^{\prime \prime}$ \\
\hline 4) Flexible rotor blades & $36^{\prime \prime}$ \\
\hline
\end{tabular}




\section{RIGID RoTOR BLADES}

Since the motion near the blade tip was faster than in the cantilevered beam experiment a lower exposure time was required from the cameras, which meant that more light was required as well. A data set consisted of a starting $0^{\circ}$ collective baseline image at the specified frequency followed by 100 images at the same frequency and a specified collective pitch angle. An example data set might be one image of $0^{\circ}$ collective at 1500rpm, followed by 100 images at $5^{\circ}$ and $1500 \mathrm{rpm}$.

\section{4" Diameter Rotor}

For the 24" diameter rotor the entire blade could not fit inside the field of view of the cameras and caused the results for this experiment to be offset by approximately 3.5 " from the root of the blade. In order to get the blade at the proper azimuth position a time shift $(\Delta \mathrm{t})$ was implemented that would offset from the input trigger before capturing an image frame; however, since the rotor frequency fluctuated slightly over time the blade's azimuth position relative to the camera would appear to fluctuate as well, this fluctuation was greater with larger time shifts. This made it so that a standard mask as used previously could not be applied; instead an algorithmic mask was utilized that masked out pixels that did not have a minimum intensity count.

As mentioned previously, the full-field vector deformation was calculated at two rotational speeds $(1500$ and $1800 \mathrm{rpm})$ and five pre-set collective pitch angles $\left(3.6^{\circ}, 6.1^{\circ}\right.$, $8.5^{\circ}, 12.3^{\circ}$, and $14.8^{\circ}$ ). The principal goal was to extract the bending and torsion deformation from the DIC data. The quarter-chord line was examined for the bending mode shape while the slope of the chord line was used to find the twist angle at each point along the span. The deformation results were fairly consistent with negligible variation between set vector fields, though outliers did exist. The mean value of deformation between all vector fields was calculated at each span-wise location while 
excluding the upper and lower $10 \%$ of outliers. The mean lines produced in this way had smoother mean curves than if all the data were included in the calculation. The following figures help to illustrate the results for a sampling of data at $1800 \mathrm{rpm}$ and 8.5 degree collective pitch; the spatial coordinates are given as a fraction of the blade length and the pitch is displayed as a fraction of the inclinometer collective pitch.
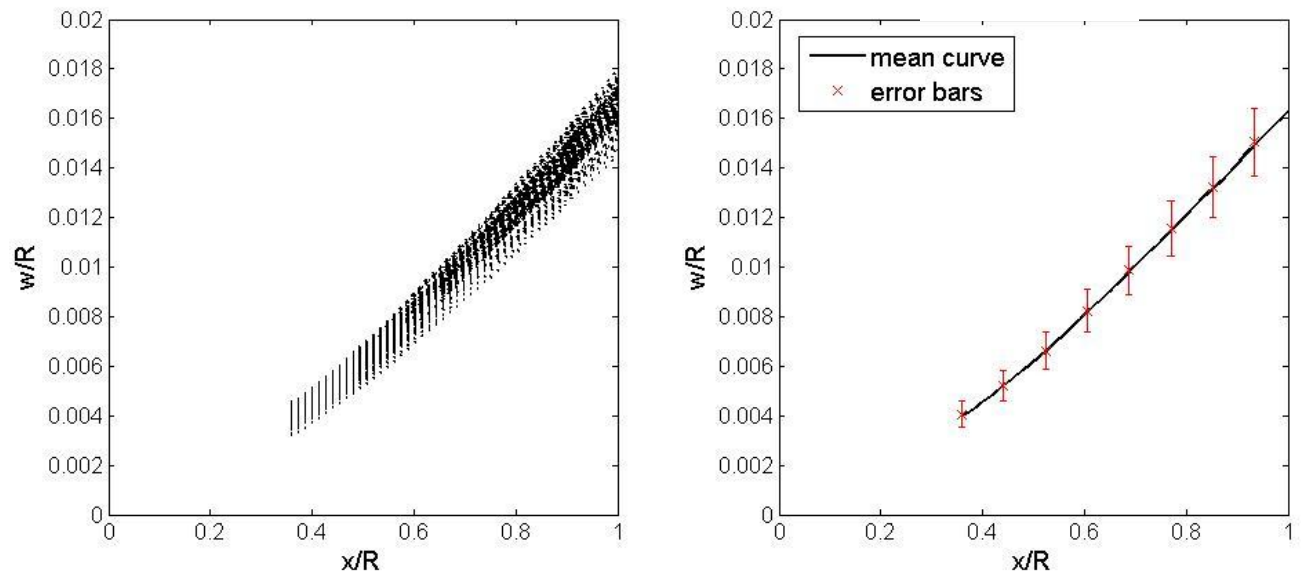

Figure 5.1.1: Raw deflection data for all images in a set for 24" diameter rigid blades; $1800 \mathrm{rpm}$ at 8.5 degree collective pitch (left) and mean deflection with error bars (right)

The deflection profile for the rigid blades shows on the order of what was expected; a nearly linear curve with subtle bending primarily near the root of the blade. The magnitude of deformation is very small relative to the length of the blade and the rigid flap angle can be extracted from the arctangent of the slope, in this case about 0.135 degrees.

The pitch was calculated at each span-wise location by considering $2 \mathrm{D}$ chordwise sections of the blade and locating two points at the leading and trailing edges of the blade as shown in figure 5.1.2, then the pitch is simply the arctangent of the slope of a 
line connecting the two points. However, it should be understood that this method relies on having blade surfaces that are perfectly horizontal (or vertical) in the camera view window; should the blade be rotated the slightest amount from the horizontal axis, then the chord length will appear to be faintly greater and will result in minor pitch undercalculations (see fig. 5.1.3). If the azimuth position does not change between camera images then adjustments can be made to appropriately resolve the issue.

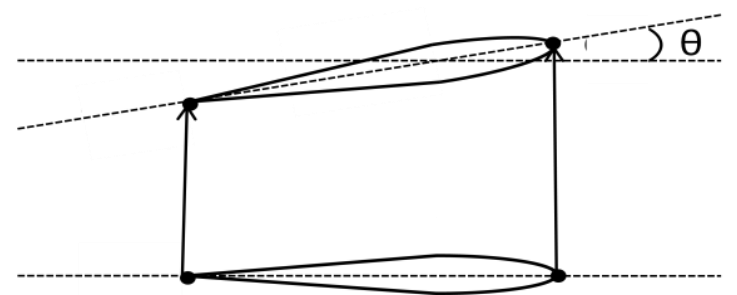

Figure 5.1.2: Illustration for the method of sectional pitch calculation

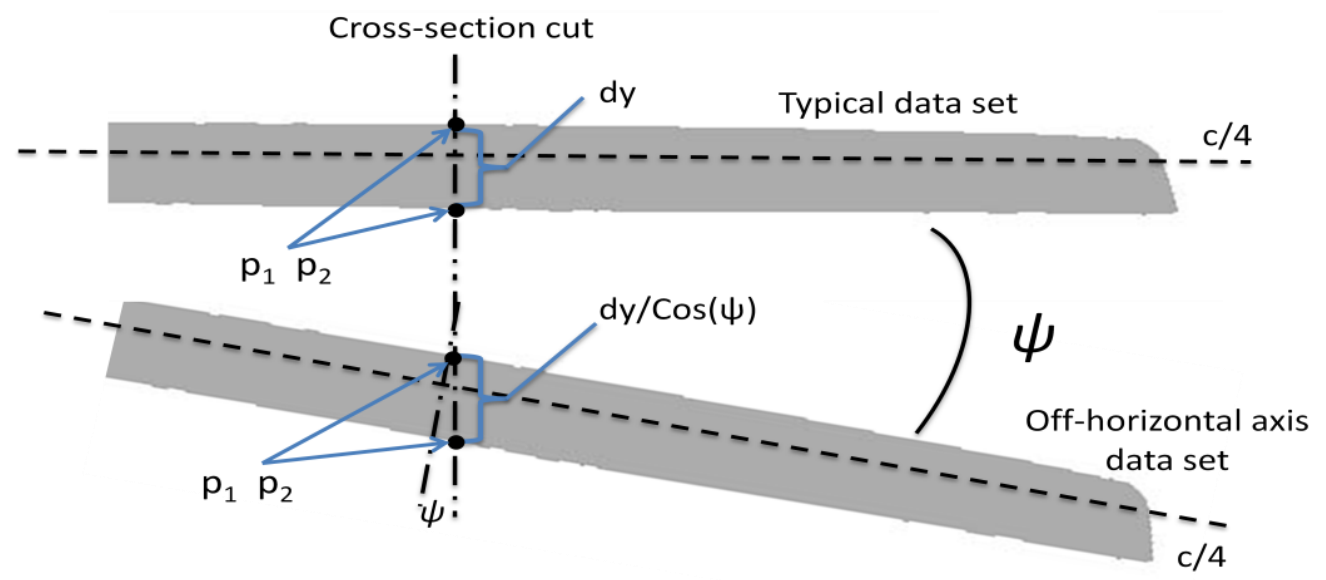

Figure 5.1.3: Difference between a typical and an off-horizontal data set illustrating the change in measured chord length 

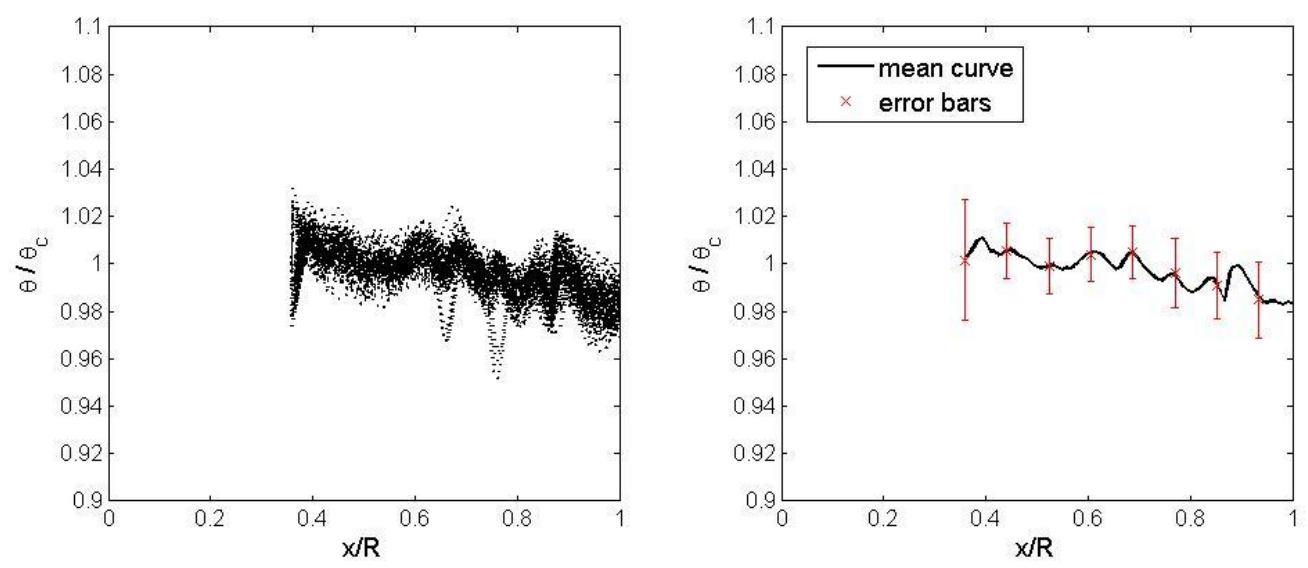

Figure 5.1.4: Raw pitch distribution data for all images in a set for 24" diameter rigid blades; $1800 \mathrm{rpm}$ at 8.5 degree collective pitch (left) and mean pitch distribution with error bars (right)

As expected from the rigid blades, the span-wise variation in pitch (fig. 5.1.4) was small and the mean curve was roughly constant; however, the pitch was always measured at slightly different values than the input collective. Additionally, the slight scattering of pitch values at each radial position was also observed by Olson et.al. ${ }^{16}$ in their calculation of pitch on a full-scale UH-60A rotor blade, it was concluded that this variation was possibly due to imperfections in the airfoil lower surface geometry. The error associated with twist is further propagated because twist is a derived quantity from multiple displacements. In this case the pitch angle was calculated at approximately $95 \%$ of the input collective with a standard deviation of 5\%. The relatively large standard deviation in the pitch calculation is due to the observed fluctuation of the blade azimuth position relative to the camera view window as previously discussed. This variation tended toward the clockwise of horizontal and resulted in a slight under-calculation of pitch.

The estimated accuracy in the deflection should be about 0.05 pixels, as determined by the size of the interrogation window and a Lavision look-up table. Given that the calibration factor for this experiment was 198.7 pixels/in and the length of the 
beam was 9.8 " we can calculate the expected error at $0.00257 \% \mathrm{R}$; however, the mean standard deviation observed was approximately $0.0387 \% \mathrm{R}$ or 0.0038 degrees. The mean error in the theta calculation was higher at approximately 0.1055 degrees. This indicates that there is some other factor that is reducing the repeatability of the results. In fact, each of the curves seem to have approximately the same shape with some linear shift in the y-axis data, this implies that there is some minor inconsistency in the rotor hub from one revolution to another; vibration of the support structure for example.

As expected, an increase in collective pitch angle results in greater flap-wise deformation (see fig. 5.1.5). More interesting, as can be seen in figure 5.1.6, is that at higher collective pitch angles the mean pitch curves appeared to have a slight negative slope indicative of some small negative elastic twist.

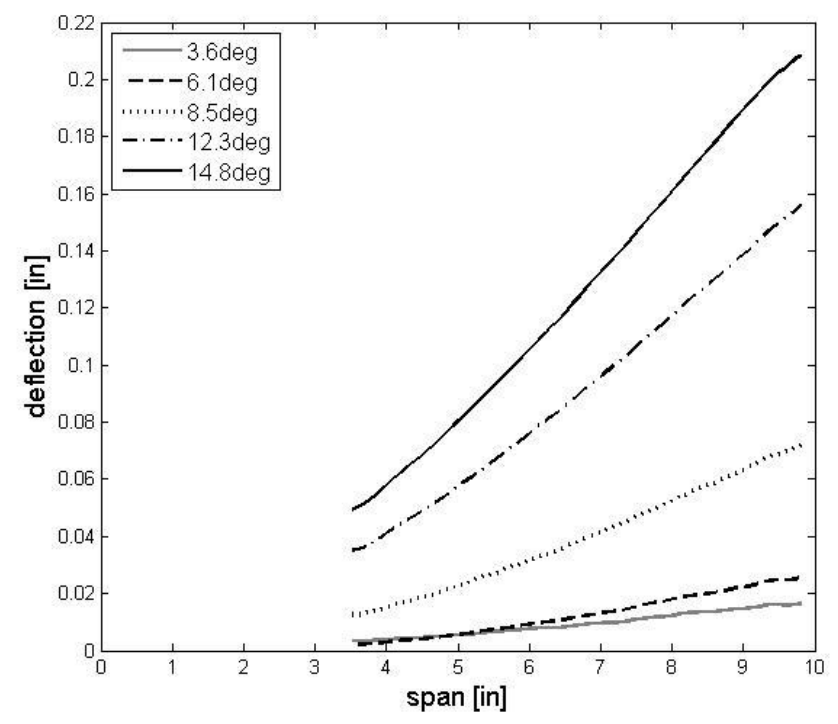

Figure 5.1.5: Mean deflection curves for 24" diameter rigid blades at $1500 \mathrm{rpm}$; various collective pitch angles 


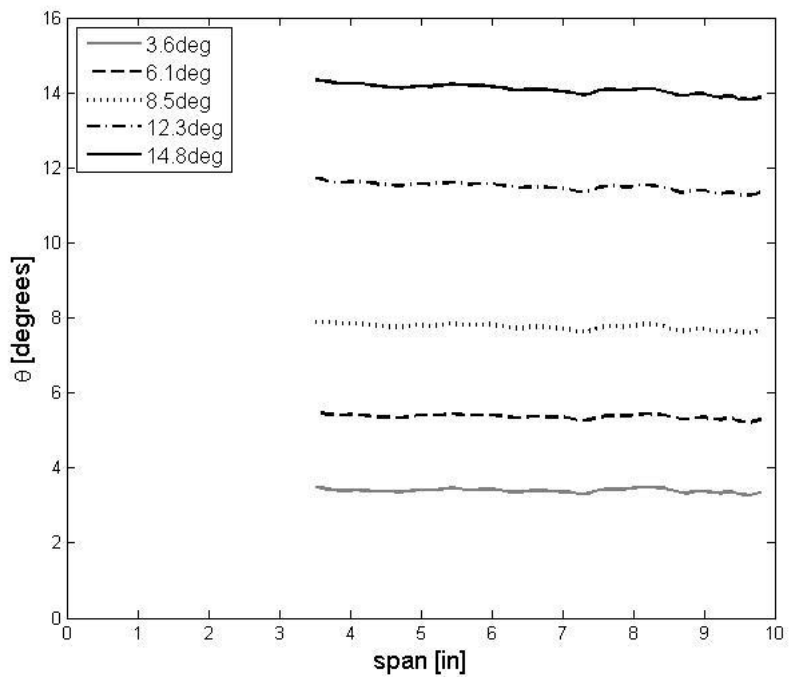

Figure 5.1.6: Mean pitch distribution curves for 24" diameter rigid blades at 1500 rpm; various input collective pitch angles

At $1800 \mathrm{rpm}$ the results were similar to those from 1500rpm with the exception that the magnitude of the deflection was greater. As illustrated in figure 5.1.7 and 5.1.8 respectively, the magnitude of deflection and the rigid flap angle are greater with both rotor speed and collective pitch angle. Additional deflection results at 1800rpm are given in the appendix (Fig. A1).

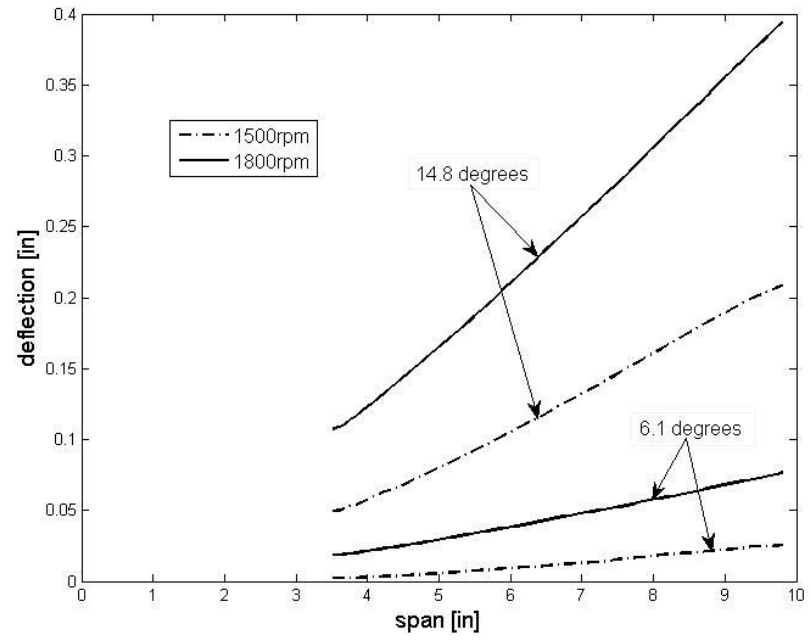

Figure 5.1.7: Mean deflection curves for 24" diameter rigid blades at $1500 \mathrm{rpm}$ and $1800 \mathrm{rpm} ; 6.07^{\circ}$ and $14.76^{\circ}$ collective pitch angles 


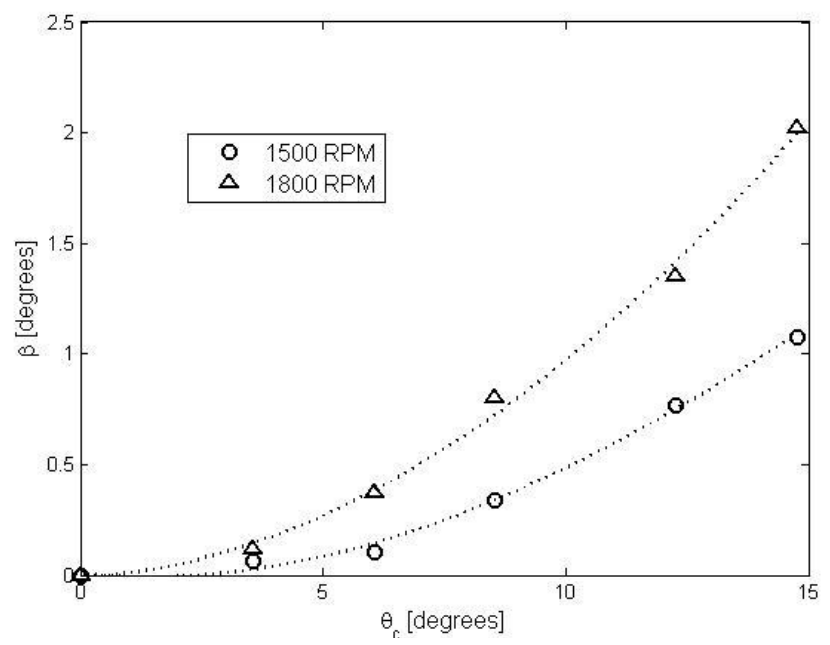

Figure 5.1.8: Rigid flap angle vs. collective pitch angle for 24" diameter rigid blades at $1500 \mathrm{rpm}$ and $1800 \mathrm{rpm}$

The results of the BEMT algorithm were relatively comparable to load cell measurements. The thrust measurements taken concurrently with the DIC data were faulty and archived thrust measurements at 1800rpm for the same blades were used for comparison purposes. The difference between the load cell thrust measurements and the BEMT approximations could be due to the paint on the lower surface of the airfoil changing the airfoil characteristics as well as the approximate airfoil coefficients used at a Reynolds number other than the actual test condition. The lift and drag coefficients for the NACA0014 airfoil (see figs. A2 and A3 in the appendix) were calculated with the JavaFoil $^{24}$ application which determines coefficients based on airfoil geometry, Reynolds Number, and angle of attack. The calculation uses $2^{\text {nd }}$ and $3^{\text {rd }}$ order panel methods to create velocity distributions along the surface of the airfoil and an integral boundary layer method to calculate friction drag. Experimental validation of the application showed that the numerical method generally over-predicts lift coefficients after the onset of stall, though its results are comparable to other numerical applications like $X f_{o i l}{ }^{25}$. To account for the lack of an appropriate stall model, the curve after stall was modified to resemble 
the shape of experimental NACA0012 lift curves ${ }^{27}$ (note that NACA0014 experimental lift curves were not available). At higher collective pitch there is greater error in the pitch calculation, as shown in figure 5.1.9, which results in a progressive undercalculation of thrust; the thrust curve is seen to deviate slightly with increasing collective from the BEMT approximation for constant blade element pitch, as shown in figure 5.1.10; before stall the deviation is always within $12 \%$. The rotor thrust coefficients for the DIC data calculated from BEMT are approximately the same for both rotor speeds, as expected (see fig. 5.1.11). At lower collective there is negligible difference in the thrust coefficient between the constant blade element pitch results and the DIC results calculated with BEMT, at higher collective the small difference can be attributed to pitch measurement error as previously discussed.

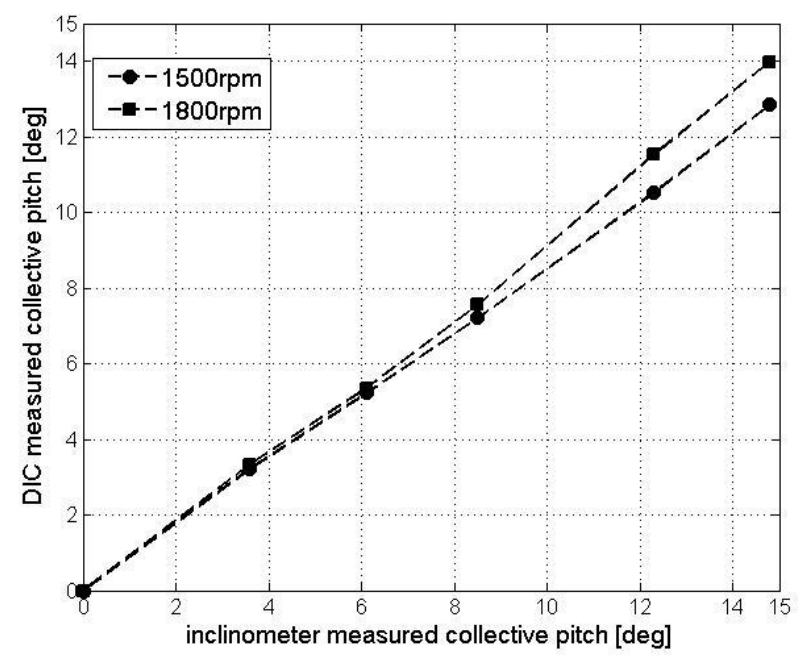

Figure 5.1.9: Inclinometer measured collective pitch vs. DIC measured collective pitch for both 1500 and $1800 \mathrm{rpm}$ 


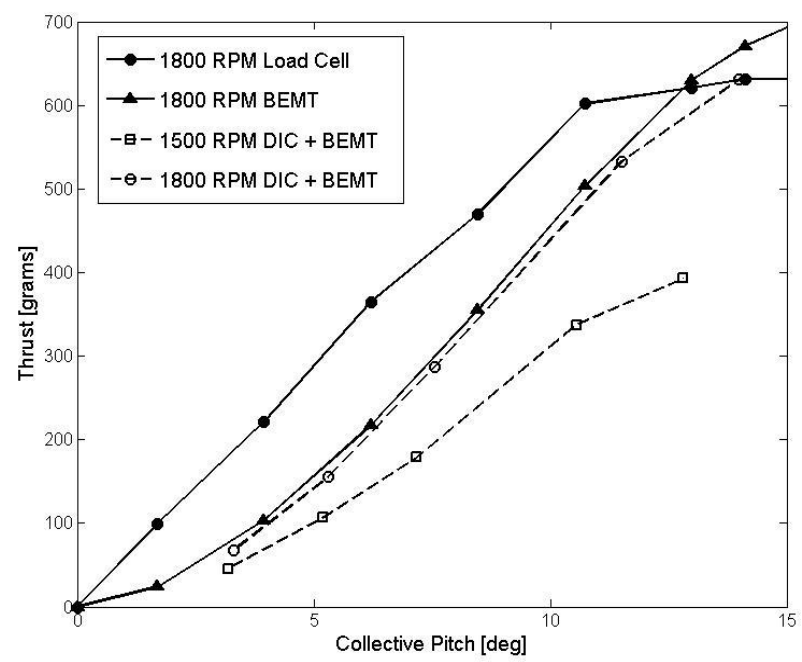

Figure 5.1.10: Thrust vs. collective pitch angle, comparison between DIC + BEMT results and load cell measurements.

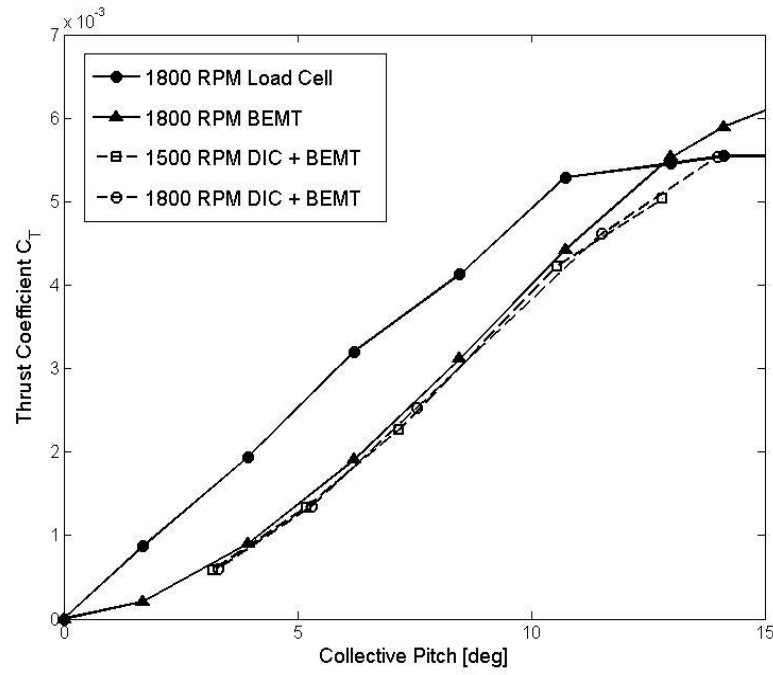

Figure 5.1.11: Thrust coefficient vs. collective pitch angle, comparison between DIC + BEMT results and load cell measurements.

Knight \& Hefner ${ }^{23,26}$ demonstrated that theoretical BEMT approximations for power are generally in good agreement with experimental results although somewhat 
over-predicted, particularly at higher rotor solidities. The rotor in this experiment has a solidity of approximately 0.0463 , which is relatively low; as can be seen in figure 5.1.12 the DIC approximations are in close agreement with the load cell measurements, although slightly over-predicted, as expected. Interestingly, the DIC approximated power is closer to the true power than the approximation with constant blade element pitch; this is because the pitch angle is under-calculated from the DIC data and there is also some minor elastic twist.

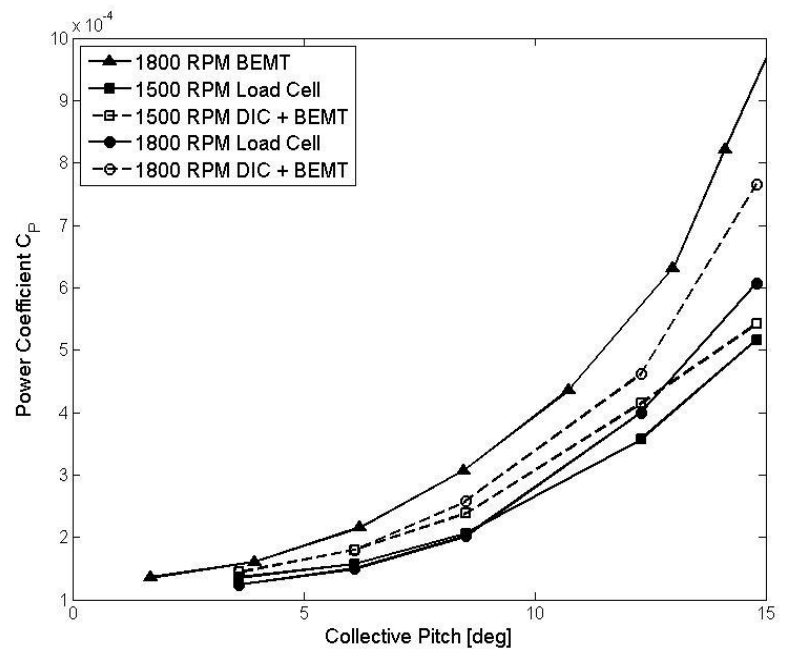

Figure 5.1.12: Power coefficient vs. collective pitch angle, comparison between DIC + BEMT results and load cell measurements.

The thrust and power relationship (fig. 5.1.13) is approximately the same between the BEMT approximations and the load cell measurements. The disparity between the DIC and constant blade pitch results on the figure of merit curve (fig. 5.1.14) is due to the difference in power since the thrust coefficients are nearly the same; after 12.3 degrees the DIC results are greater than the constant pitch results, this is due to the decrease in thrust coefficient determined by DIC and is expected at higher collectives. The dashed 
lines in the figures correspond to an approximate best-fit to the DIC data (1500 and 1800 rpm) and the BEMT approximation for a constant blade element pitch respectively.

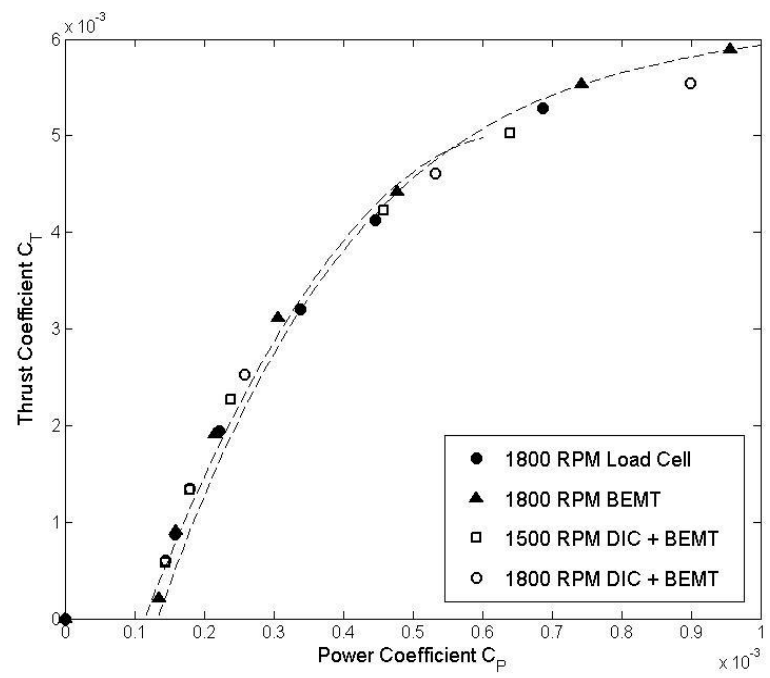

Figure 5.1.13: Thrust coefficient vs. power coefficient, comparison between DIC + BEMT results and load cell measurements

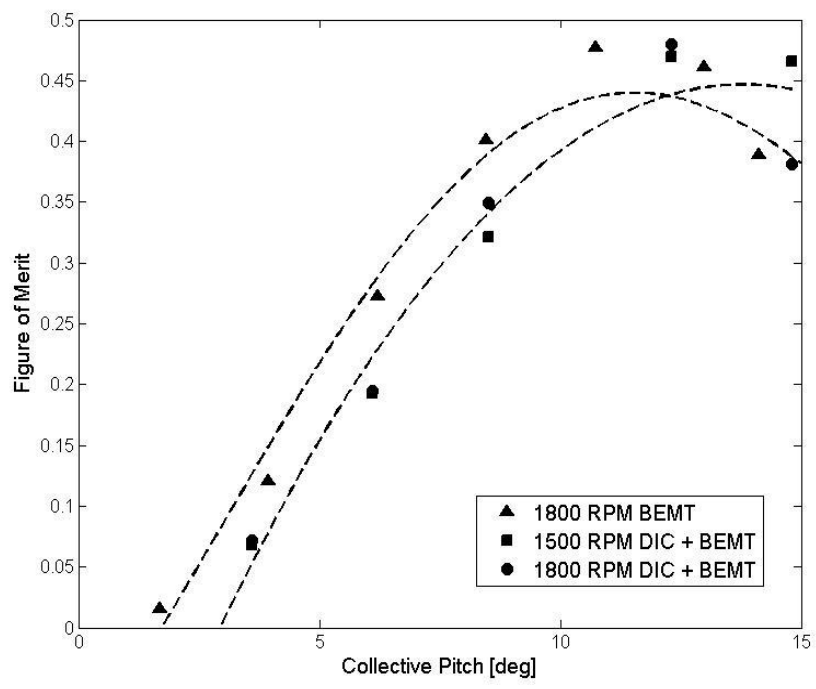

Figure 5.1.14: Figure of Merit vs. collective pitch angle, comparison between DIC approximation and constant blade element pitch approximation 


\section{9" Diameter Rotor}

For the 39" diameter rotor the results are offset by approximately $7 "$ from the root of the blade due to the entire blade being too large to fit inside the field of view of the cameras. Also, due to the use of a strobe light for lighting there was no need for a phase delay and the fluctuation of the rotor azimuth position was eliminated, though an algorithmic mask was still employed for its simplicity.

As expected of the rigid blades, the flap-wise deflection curves are quite linear (see figs. 5.2.1 \& 5.2.2). As was the case with the 24" diameter rigid blades, an increase in rotor speed or collective pitch results in greater deflection.

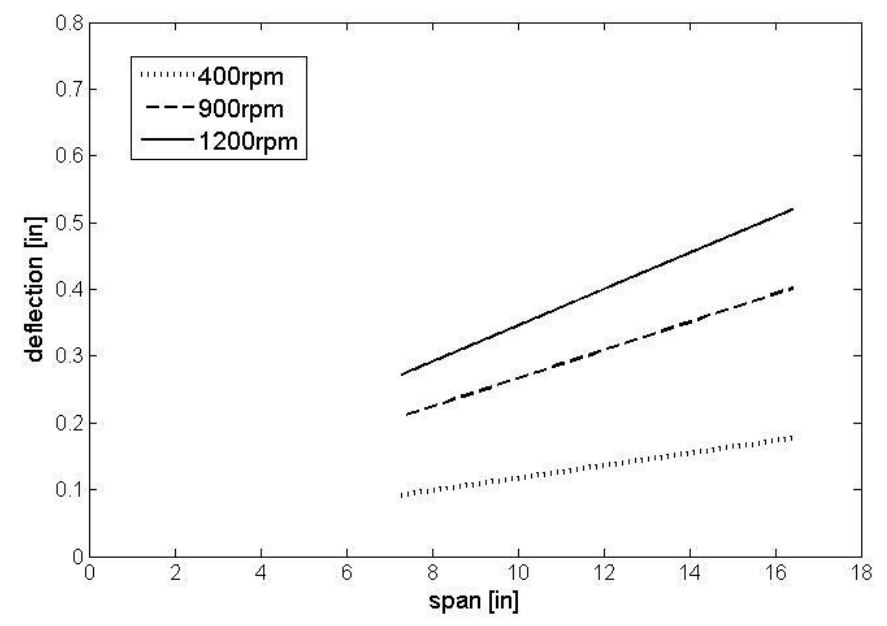

Figure 5.2.1: Mean deflection curves for 39" diameter rigid blades at 4.5 degree collective 


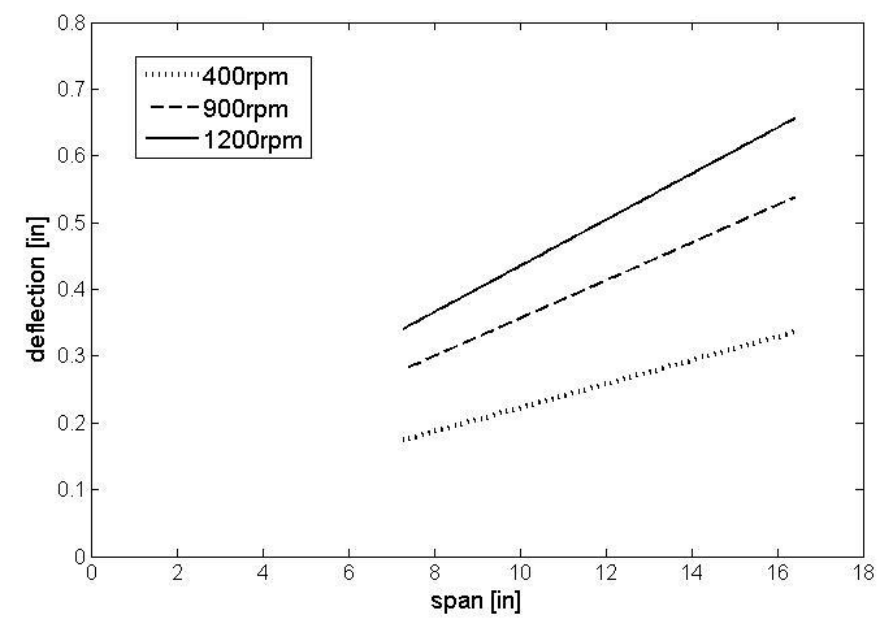

Figure 5.2.2: Mean deflection curves for 39" diameter rigid blades at 8 degree collective

The blades are reasonably rigid in twist, though there is a clear trend in all of the data in which the pitch near the tip of the blade is less than the pitch closer to the root; in other words there exists a small degree of negative twist along the span, as can be seen in figures 5.2.3 and 5.2.4. If the blades were more flexible it is assumed that the twist would be larger in magnitude. Adjustments to the rotor hub between measurement sets also caused small changes in the collective pitch. Additionally, as discussed previously, the slight scattering of pitch values at each radial position is due to imperfections in the airfoil lower surface geometry which was also observed by Olson et.al. ${ }^{16}$ in their calculation of pitch on a full-scale UH-60A rotor blade. 


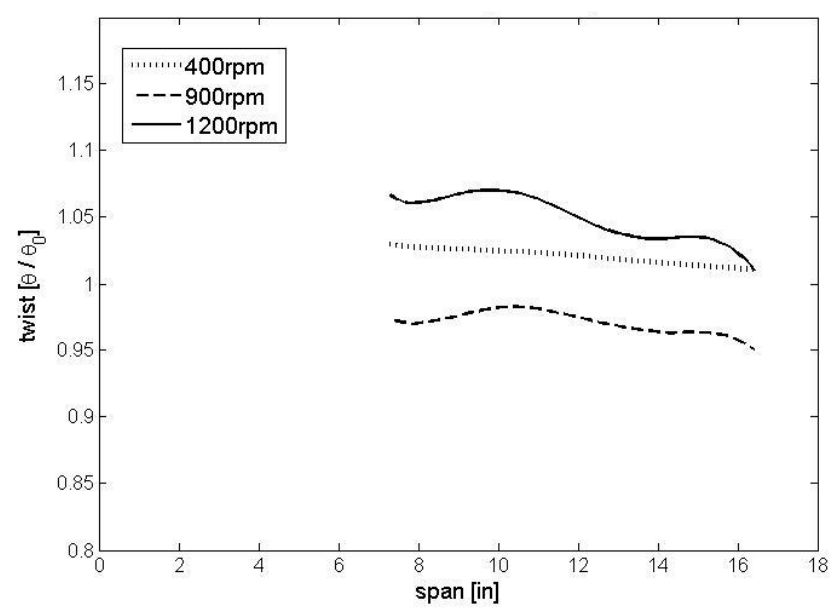

Figure 5.2.3: Mean twist curves for 39" diameter rigid blades at 4.5 degree collective

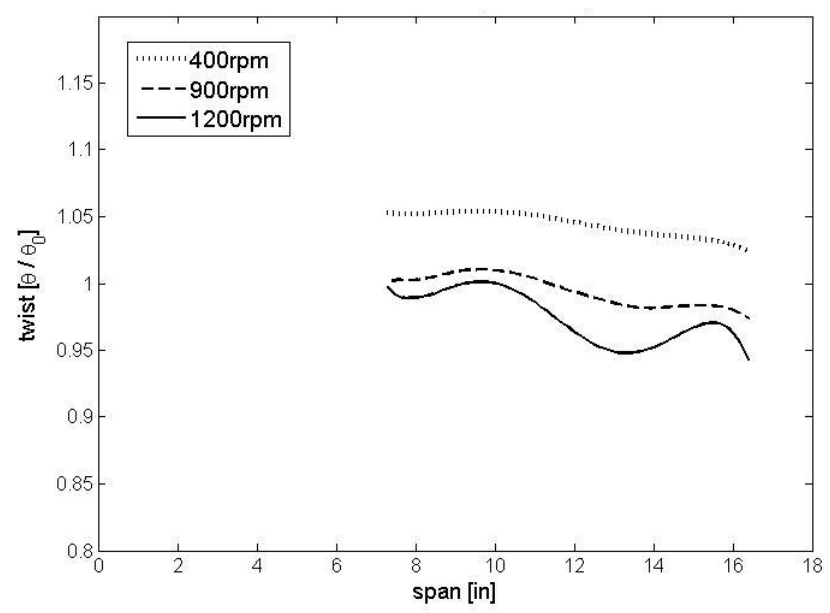

Figure 5.2.4: Mean twist curves for 39" diameter rigid blades at 8 degree collective

As anticipated, the rigid flap angle (or coning angle) increases with increased rotor speed or collective pitch. The relationship between coning angle and rotor speed appears to be linear while an increase in collective pitch provides a vertical shift of this line and maintains its slope (Fig. 5.2.5). Data at a wider range of collective pitch is necessary to provide a more clear correlation between coning angle and collective. Note 
that the first measurement is at 400rpm, the lack of resolution in rpm (particularly from 0400rpm) causes there to appear to be a discontinuity in the 8 degree collective curve, though the true shape of this curve would likely approach zero differently.

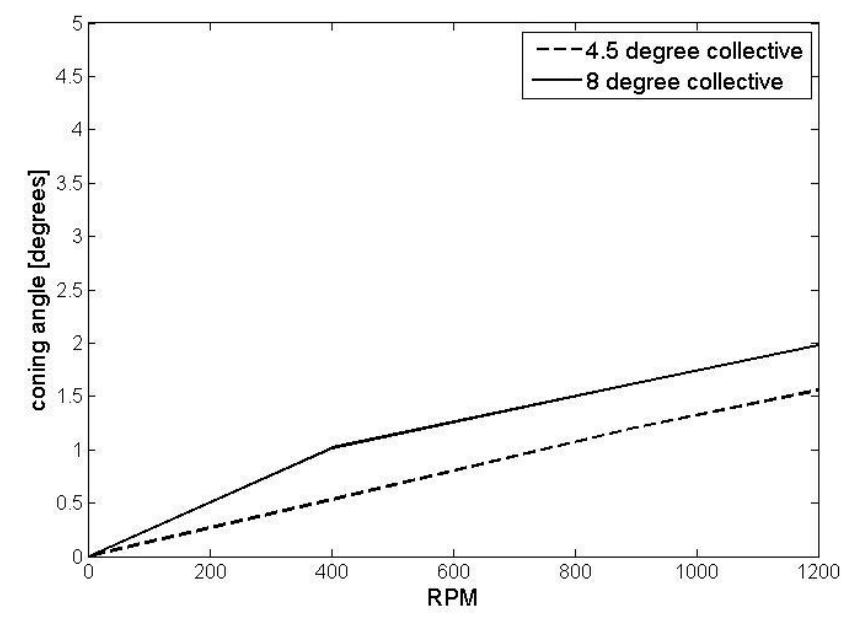

Figure 5.2.5: Rigid flap (coning) angle vs. rpm for 39" diameter rigid blades

Utilizing the same BEMT routine that was used on the 24 " rotor blades the thrust was approximated (see Fig. 5.2.6); although in this experiment load cell data was not available to compare to, it is assumed that the thrust is slightly under-calculated, as was the case in the previous experiment. This helps to illustrate a point in that DIC allows for the approximation of loading even when more exact measurement methods are unavailable. The increase in thrust with both rpm and collective pitch is as expected. The thrust and power coefficients, and figure of merit, were relatively constant across all rpm tested, only increasing significantly with collective pitch (see Table 5.2).

Table 5.2: Variation of thrust and power coefficients, and figure of merit, with collective pitch for 39" diameter rigid rotor

\begin{tabular}{|c|c|c|c|}
\hline$\theta_{\mathrm{c}}$ & $\mathrm{C}_{\mathrm{T}}$ & $\mathrm{C}_{\mathrm{P}}$ & $\mathrm{FM}$ \\
\hline $4.5^{\circ}$ & 0.0017 & 0.00037 & 0.1316 \\
\hline $8^{\circ}$ & 0.0043 & 0.0006 & 0.3358 \\
\hline
\end{tabular}




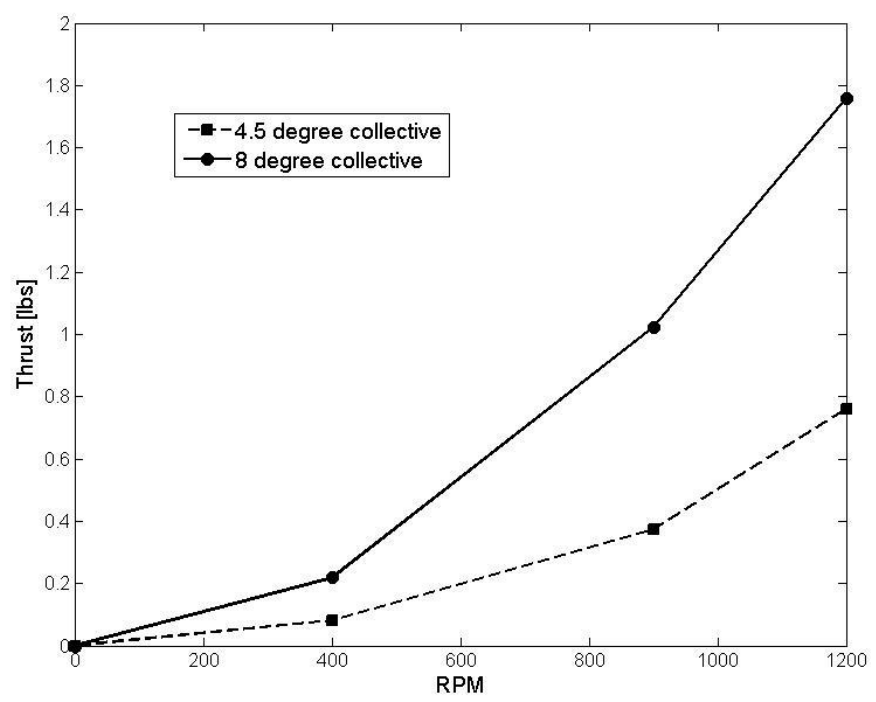

Figure 5.2.6: Predicted thrust vs. rpm for 39" diameter rigid blades using span-wise variation in pitch angles measured by DIC in conjunction with BEMT

\section{FLEXIBLE ROTOR BLADES}

Once again as can be seen in this section, the pitch angles calculated were often slightly underestimated due to slightly off-horizontal blade surfaces. Also, in a number of cases the vector deformation field was not calculated correctly and went to zero, these lower the mean deflection and pitch angle curves, though in the following results those outliers were eliminated prior to calculation. Figure 5.3.1 shows that of the 3 flexible blades, blades $\mathrm{C} 4$ and BP produce noticeably more thrust than blade $\mathrm{CF}$, with blade BP being the most effective. Blade BP would be more effective if it were not for the weight of the tip mass, and blade CF actually produces some negative lift at the location of the flap, reducing the net thrust over the blade. The following sections will further explain this trend. 


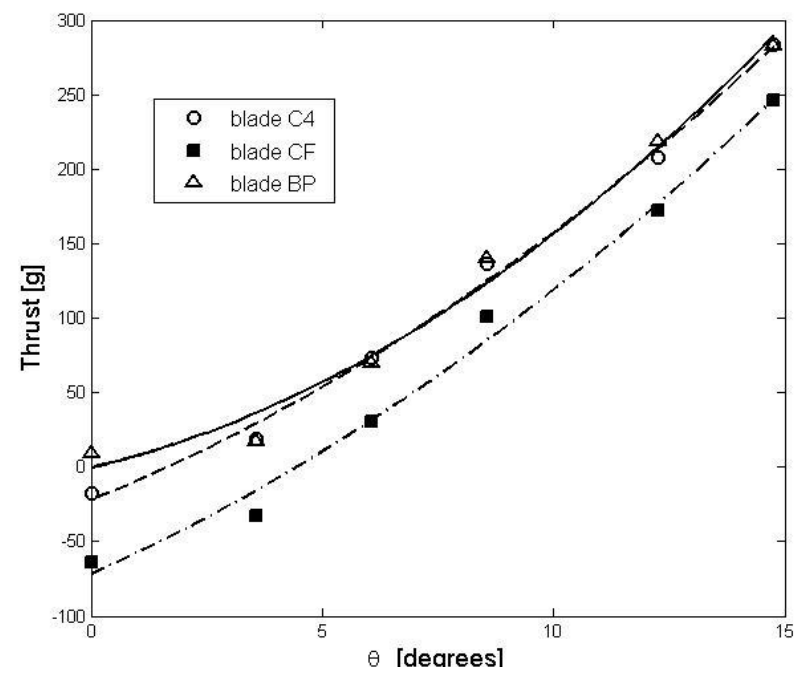

Figure 5.3.1: Thrust vs. collective pitch angle for each of the flexible blades at 1800rpm

\section{Baseline Blade C4}

The bending (flap-wise) deflection of the blades is significantly larger than in the case of the rigid blades as can be seen from figure 5.3.2. At low thrust levels (low collective) the bending is distributed over the blade span, while at higher thrust levels most of the bending is localized near the blade root forming a virtual flap hinge. Figure 5.3.3 shows the twist distribution along the blade span. The y-intercept of the curves gives the collective pitch angle, and it is seen that these are lower than the input (actual) values. The discrepancy between measured and actual collective pitch increases with increasing collective pitch; the reason for this discrepancy is that the pitch is calculated from points located at the leading and trailing edges, but the leading edge point cannot be determined exactly due to the fact that the leading edge is obscured from the view of one camera, to a greater extent at higher collectives. For this reason, the leading edge point is actually the point closest to the leading edge. 


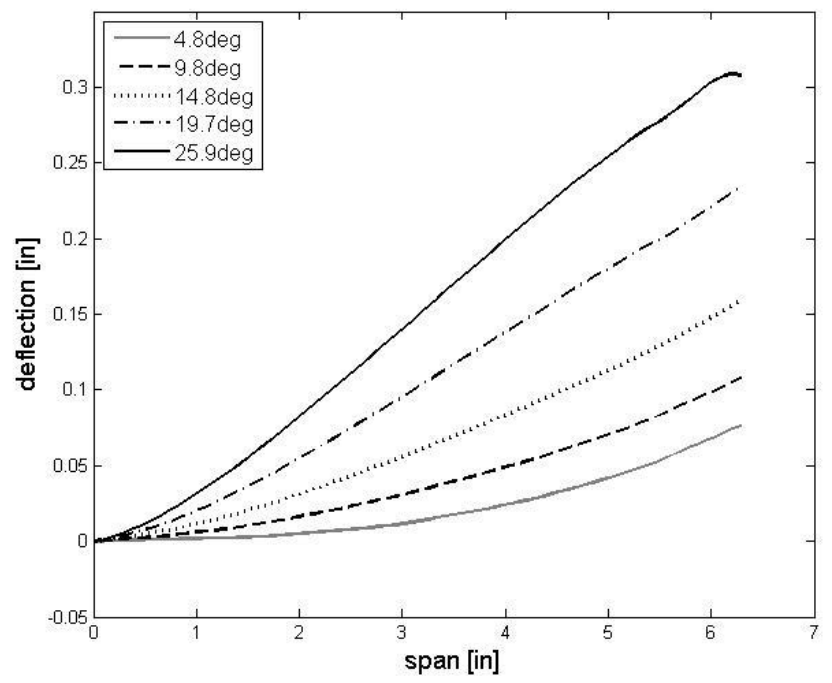

Figure 5.3.2: Mean deflection curves for blade $\mathrm{C} 4$ at various collective pitch

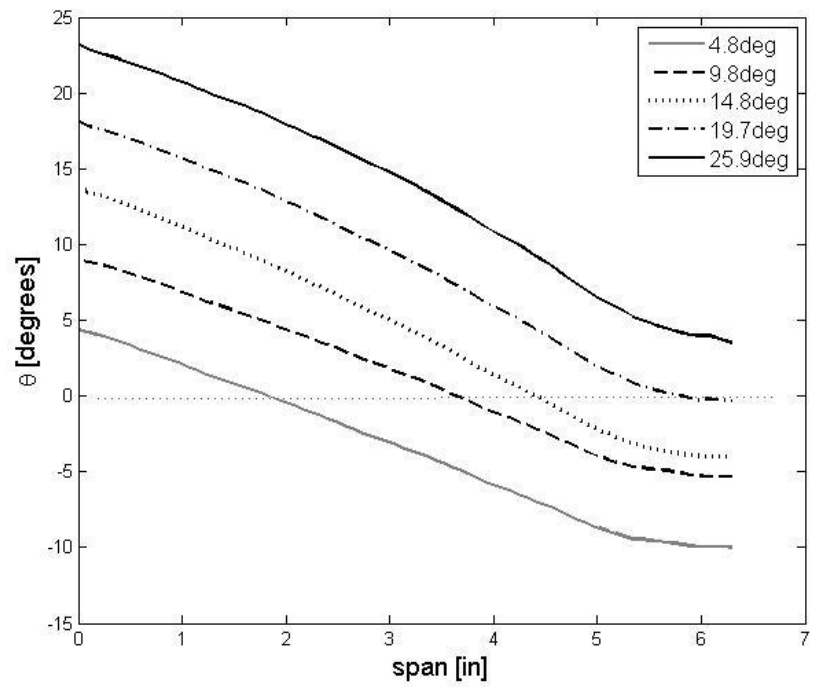

Figure 5.3.3: Mean pitch distribution curves for blade $\mathrm{C} 4$ at various collective pitch

The span-wise distributions of twist and bending yield enormous insight into the physics of the flexible blades. Note that these measurements would have been extremely difficult with conventional techniques. From figure 5.3.3 it is also seen that the flexible blades $\mathrm{C} 4$ have a high negative twist, such that at low collective pitch the blade tips have 
a negative incidence. This negative twist is induced by the propeller moments on the blade tip and is responsible for the poor figure of merit of this rotor (see Sicard and Sirohi $^{21,22}$ ). The blade designs CF and BP were developed to alleviate this negative induced twist. Figure 5.3.3 also shows a decrease in the span-wise rate of twist near the blade tips. This is due to a local increase in torsional rigidity caused by a thicker layup as well as the tip mass.

\section{Blade CF}

Figures 5.3.4 and 5.3.5 show the span-wise deflection and twist respectively of the blades CF. These blades incorporate a trailing edge flap at the blade tip designed to alleviate the nose-down passive twist by creating a pitching moment. From figure 5.3.5 it is seen that the flap (deflected up) decreases the span-wise nose-down twist compared to the baseline flexible blade $\mathrm{C} 4$ case (Fig. 5.3.3). The decrease in twist results in increased thrust at the same collective angle; this appears as an increase in bending deflection as seen in Fig. 5.3.4, compared to the baseline C4 case.

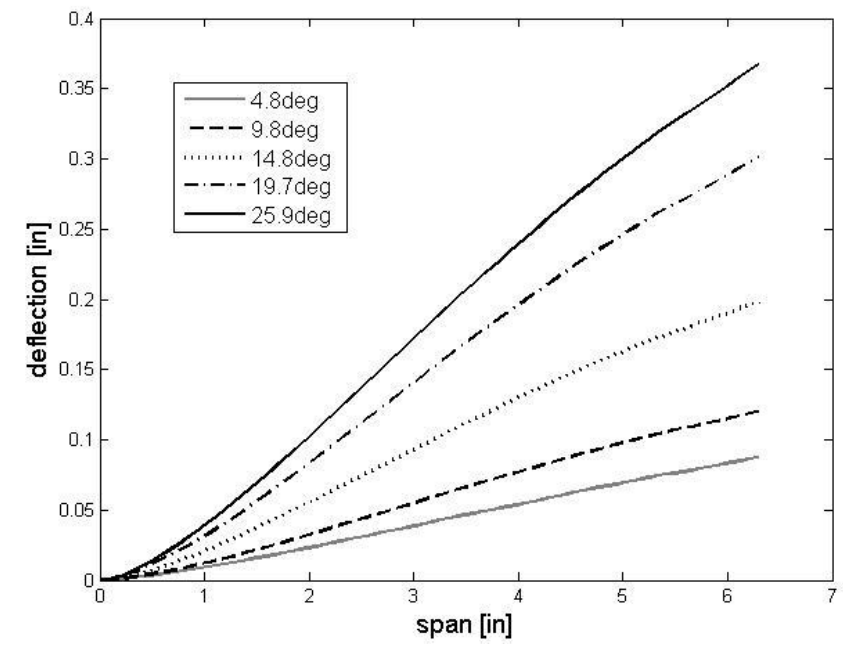

Figure 5.3.4: Mean deflection curves for blade $\mathrm{CF}$ at various collective pitch 


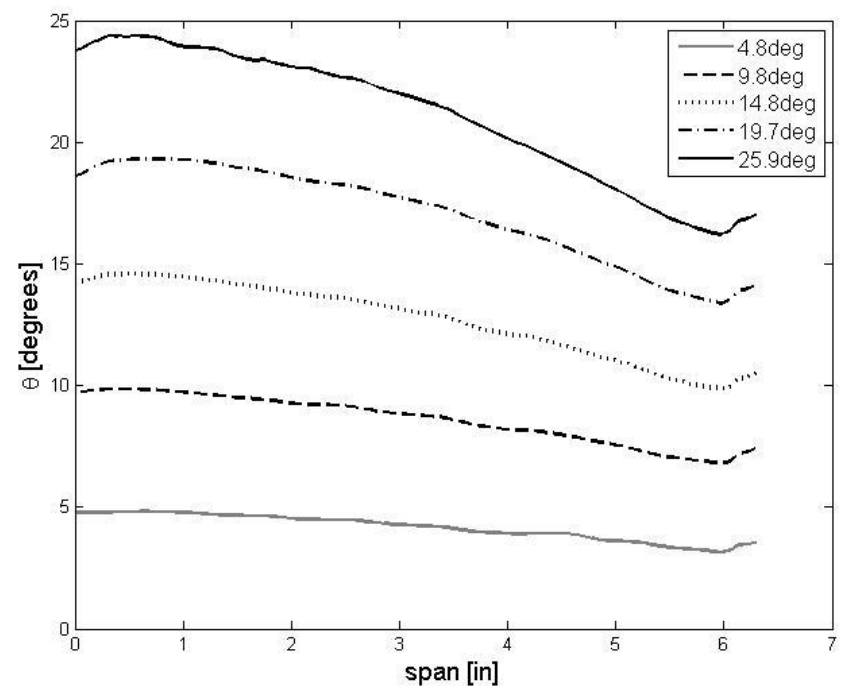

Figure 5.3.5: Mean pitch distribution curves for blade $\mathrm{CF}$ at various collective pitch

In addition, the increase in twist near the blade tip indicates that the pitching moment created by the flap results in a large local change in incidence that is not entirely transferred along the blade span due to the extremely low torsional stiffness of the blade. This increase in twist near the blade tip results in a downward force that is responsible for the decrease in bending slope near the blade tips. In general, these measurements validate the design and operation of the trailing edge flap and also indicate that the downward force near the blade tips is responsible for its reduced figure of merit (see Sicard and Sirohi ${ }^{21,22}$ ).

\section{Blade BP}

Blade BP is the most effective of the 3 flexible blades presented, as shown in figure 5.3.1 it produces the most thrust, and figure 5.3.7 shows that the pitch is nearly constant at lower angles of attack. The tip mass on blade BP is mounted at an angle of $22^{\circ}$ relative to the horizontal, the effect of which is a restoring moment that will attempt to adjust the pitch angle at the blade tip to $22^{\circ}$. As shown in figure 5.3.7, when the 
collective pitch angle is less than $22^{\circ}$ the blade has little to no twist and that which it has is positive; but, when the collective pitch exceeds $22^{\circ}$ the blade has a negative twist down to a tip pitch angle of $22^{\circ}$. This agrees with what was predicted by Sicard and Sirohi ${ }^{21,22}$.

In addition, the deflection curves shown in figure 5.3.6 have a concave down shape, opposite from blade C4. The same forces that provide a restoring propeller moment to the blade twist also pull the blade downward near the tip.

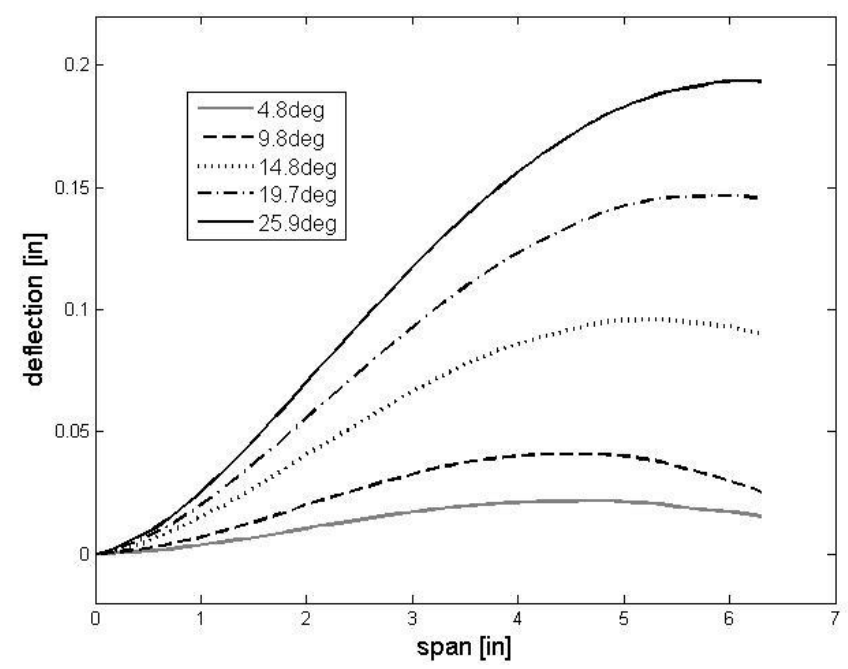

Figure 5.3.6: Mean deflection curves for blade BP at various collective pitch

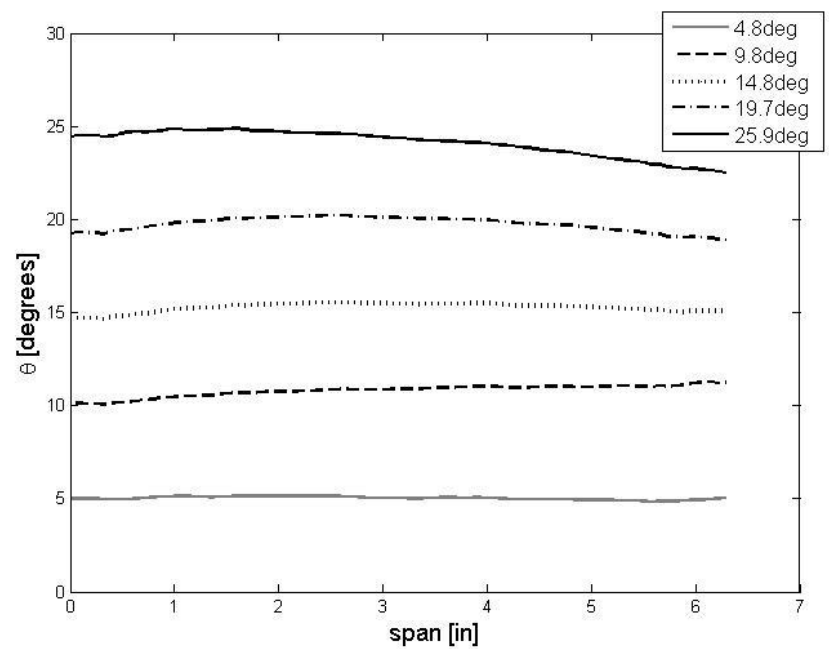

Figure 5.3.7: Mean pitch distribution curves for blade BP at various collective pitch 
Figures 5.3.8 and 5.3.9 compare all three of the flexible blades and the 24" diameter rigid rotor blades. The $\mathrm{x}$-axis in this case is span $/ \mathrm{L}$ where $\mathrm{L}$ is the length of the respective blade. The rigid blade does not extend to the root because it could not fit inside the field of view, though this could be fixed by taking a second set of measurements for a section closer to the root of the blade and merging the results as was done previously with the vibrating beam experiment.

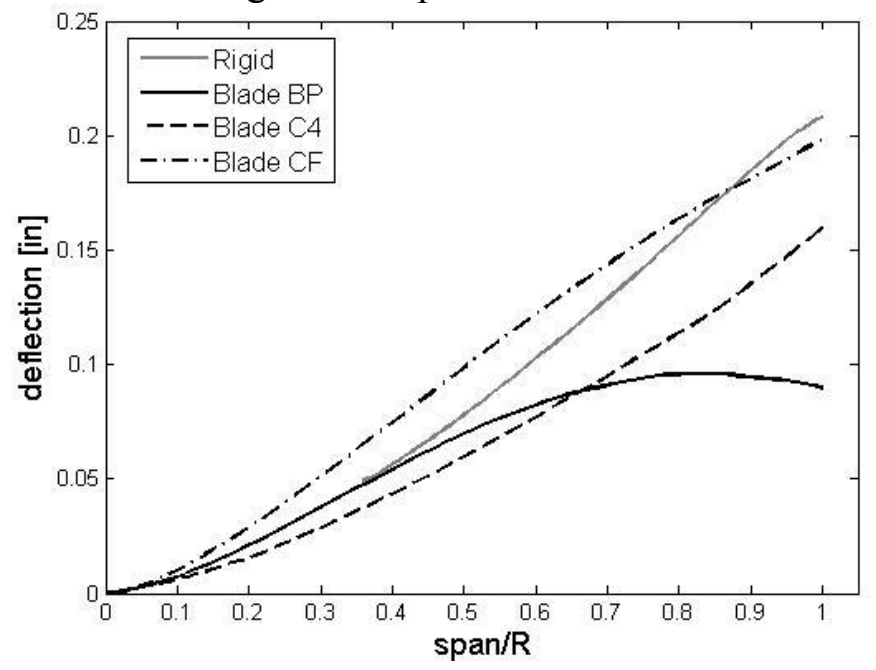

Figure 5.3.8: Mean deflection curves for various blades at $14.76^{\circ}$ collective pitch

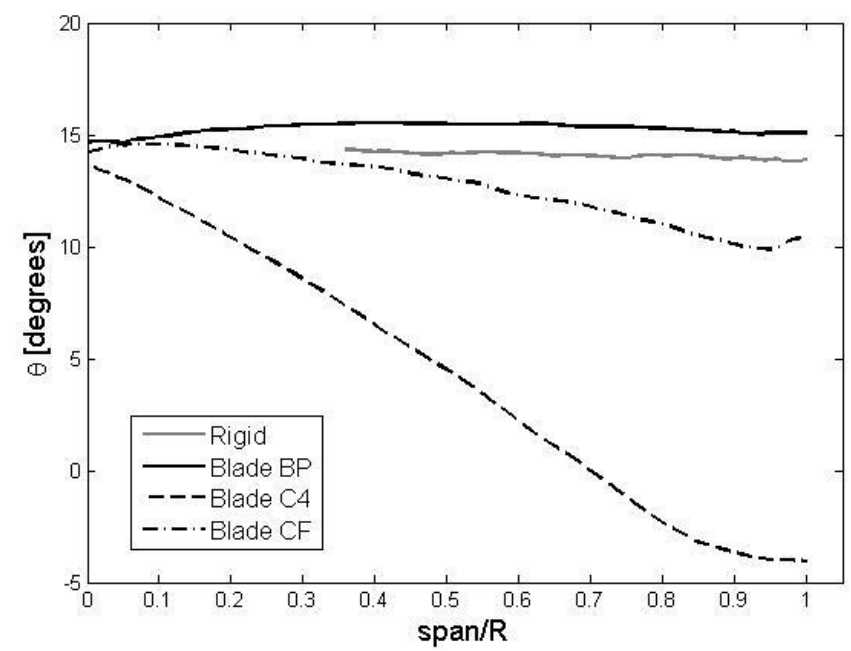

Figure 5.3.9: Mean twist distribution curves for various blades at $14.76^{\circ}$ collective pitch 


\section{Chapter 6: Conclusion}

The digital image correlation technique has proven to be a viable method for measuring the deformation of rotor blades. Flapping and pitching mode shapes are fairly easily extracted from the vector deformation field. The measured deflections have been validated in the non-rotating frame by means of measurements on a vibrating cantilever beam using a laser displacement sensor and in the rotating frame by measurements of blade pitch angle using an inclinometer. The longitudinal strain can also be calculated from the curvature of the span-wise deflection. However, the pitch angle was typically slightly under-predicted in the listed experiments due to slight blade rotation relative to a horizontal axis and obscured leading edges. The accuracy and spatial resolution of the technique enabled measurements of the flexible rotor blades which otherwise would not be possible with other techniques. The calibration factor, beam/blade length, spatial resolution, and accuracy of each of the set of experiments are tabulated in table 6.1.

Table 6.1: Accuracy Estimations of Experiments

\begin{tabular}{|l|c|c|c|c|c|c|}
\hline \multicolumn{1}{|c|}{ Experiment } & calibration factor & $\mathrm{R}$ & \multicolumn{2}{|c|}{ spatial resolution } & \multicolumn{2}{|c|}{ accuracy } \\
& (pixel/in) & (in) & (in) & $(\% \mathrm{R})$ & (in) & $(\% \mathrm{R})$ \\
\hline Vibrating beam & 243.4 & 17.8 & 0.066 & 0.369 & $2.05 \mathrm{E}-04$ & 0.0012 \\
\hline 24" Rigid rotor blades & 198.7 & 9.8 & 0.081 & 0.822 & $2.52 \mathrm{E}-04$ & 0.0026 \\
\hline 39" Rigid rotor blades & 78.2 & 16.5 & 0.204 & 1.239 & $6.39 \mathrm{E}-04$ & 0.0039 \\
\hline Flexible rotor blades & 200.8 & 6.3 & 0.080 & 1.265 & $2.49 \mathrm{E}-04$ & 0.0040 \\
\hline
\end{tabular}

The deformation profiles were used in conjunction with Blade Element Momentum Theory to calculate the thrust produced and power consumed by the rotor with results comparable to load cell measurements. At higher collective, the results indicate a tendency to under-calculate the thrust relative to constant blade element pitch results due to increased error in DIC pitch measurement at high collective. The power 
approximation is conservative relative to load cell measurements, as expected from theory; interestingly, the DIC approximation is closer to the true power than the constant blade element pitch approximation.

Some future research into making improvements to this technique may include tests on larger diameter rotors, the use of additional cameras to obtain a wider field of view and improve the accuracy of results, the use of strain gages mounted on a rotor blade to make a comparison to DIC measurements, or wind tunnel testing. There is also a demand for using the technique during live helicopter flight testing as an alternative to strain gages; the viability of the technique under those conditions should be analyzed for any potential. That is, multiple cameras mounted to a vibrating traverse or two cameras rotating at the same speed as the rotor and mounted above the hub. Additionally, it may be possible to make progressive scaling of the pitch measurements based on the collective input and airfoil geometry.

The final assessment of the technique as a method of measuring the deformation of helicopter rotor blades is that it is sufficiently accurate; even at higher collective as the error in the pitch calculation increases, the relative error in the BEMT thrust and power approximations remains small (less than $12 \%$ at $14.8^{\circ}$ collective). 


\section{Appendix}

\section{Miscellaneous Figures}

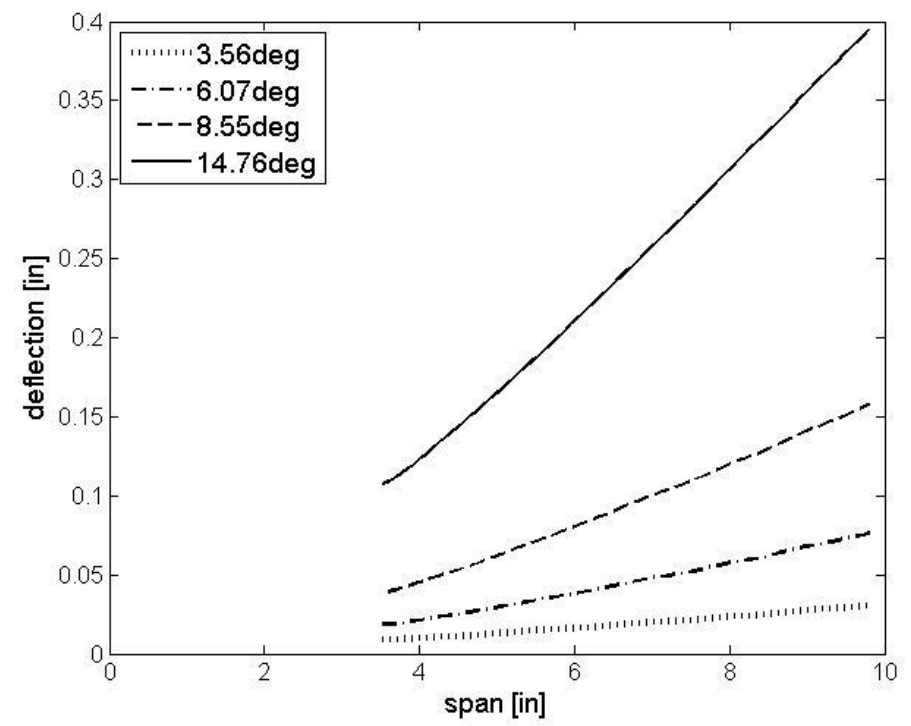

Figure A1: Mean deflection curves for 24" diameter rigid blades at $1800 \mathrm{rpm}$; various collective pitch angles

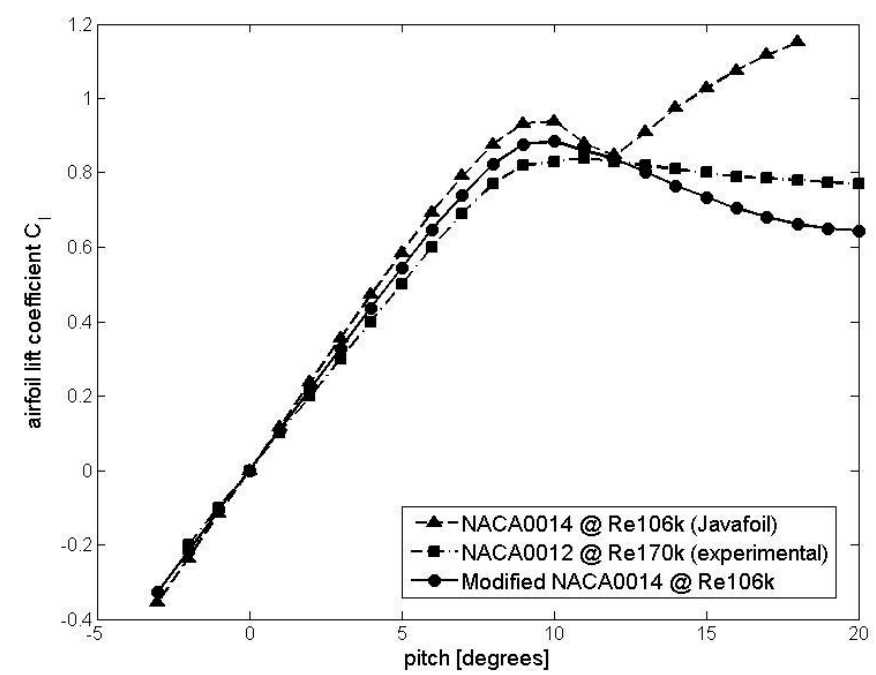

Figure A2: Airfoil lift coefficient vs. local pitch angle 


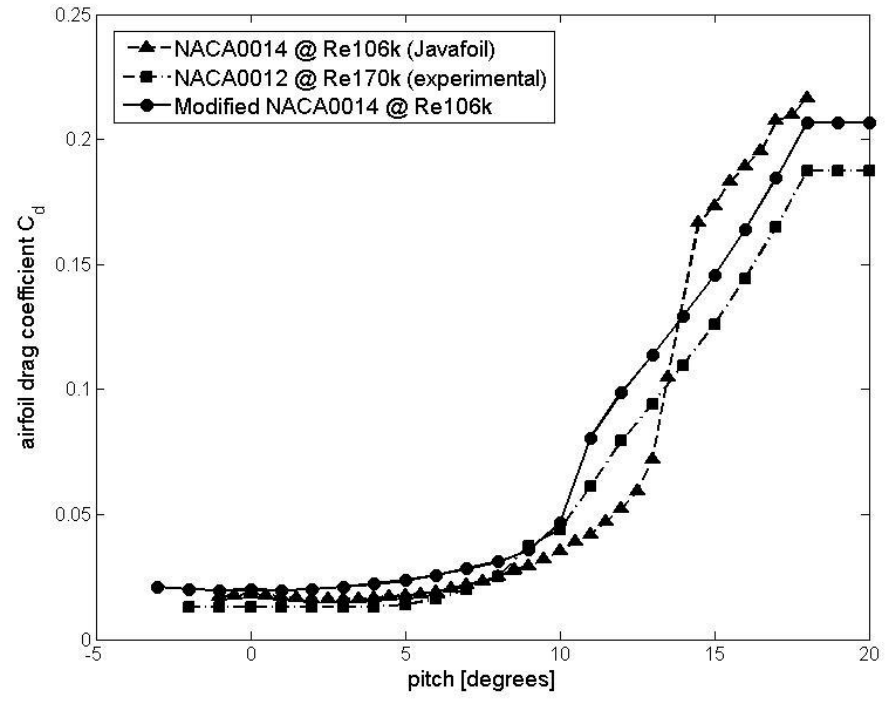

Figure A3: Airfoil drag coefficient vs. local pitch angle 


\section{References}

[1] Schmidt, T., Tyson, J., Galanulis, K., "Full-Field Dynamic Displacement and Strain Measurement Using Advanced 3D Image Correlation Photogrammetry: Part 1", Experimental Techniques, 27(3), 47-50, 2003.

[2] Rajpal S. Sirohi, Optical Methods of Measurement: Whole-field Techniques, Second edition, Francis and Taylor/CRC Press, 2009.

[3] Cloud, G. Optical Methods of Engineering Analysis, Cambridge University Press, 1995.

[4] Williams, D. C. Optical Methods in Engineering Metrology, Chapman and Hall, 1993.

[5] Tyson, J., "Noncontact Full-Field Strain Measurement With 3D ESPI", Sensors, 5, 62-70, 2000.

[6] Kahn-Jetter, Z. L., and Chu, T. C., "Three-Dimensional Displacement Measurements Using Digital Image Correlation and Photogrammic Analysis", Experimental Mechanics, 30, 10-16, 1990.

[7] Schmidt, T., Tyson, J., Galanulis, K., "Full-Field Dynamic Displacement and Strain Measurement - Specific Examples Using Advanced 3D Image Correlation Photogrammetry: Part 2", Experimental Techniques, 27(4), 22-26, 2003.

[8] Schmidt, T., Tyson, J., Galanulis, K., "Advanced Photogrammetry for Robust Deformation and Strain Measurement", SEM 2002 Annual Conference, SEM, Milwaukee, WI, June 2002.

[9] Schmidt, T., Tyson, J., Galanulis, K., "Optical Deformation \& Strain Measurements in Biomechanics", Biophotonics, 1-7, September 2003.

[10] Schmidt, T., Tyson, J., Revilock, D.M. Jr., Padula, S. II, Pereira, J.M., Melis, M., Lyle, K., "Performance Verification of 3D Image Correlation Using High-Speed Cameras", SEM 2005 Annual Conference and Exposition, SEM, Portland, OR, 2005.

[11] McNeill, S.R., Sutton, M.A., Miao, Z., Ma, J., "Measurement of Surface Profile Using Digital Image Correlation and Photogrammic Analysis", Experimental Mechanics, 30(3), 13-20, 1990.

[12] Siebert, T., Becker, T., Spiltthof, K., Neumann, I., Krupka, R., "High-Speed Digital Image Correlation: Error Estimations and Applications", Optical Engineering, 46, May 2007. 
[13] Datta, A. and Chopra, I., "Validation of Structural and Aerodynamic Modeling Using UH-60A Airloads Program Data", Journal of the American Helicopter Society, 51(1), 43-58, 2006.

[14] Steijl, R., Barakos, G. N., and Badcock, K. J., "Computational Study of the Advancing-Side Lift-Phase Problem”, Journal of Aircraft, 45(1), 246-257, 2008.

[15] Fleming, G. A., Gorton, S., "Measurement of Rotorcraft Blade Deformation Using Projection Moire Interferometry", Proceedings of the Third International Conference on Vibration Measurements by Laser Techniques: Advances and Applications, SPIE--the International Society for Optical Engineering, Ancona, Italy, June 16-19, 1998, pp. 514-527.

[16] Olson, L., Abrego, A., Barrows, D., and Burner, A., "Blade Deflection Measurements of a Full-Scale UH-60A Rotor System", American Helicopter Society Aeromechanics Specialist' Conference, San Francisco, California, January 20-22, 2010.

[17] Wu, P., Stanford, B., Ifju., P., Bowman, W., and Schwartz, A., "Digital Image Correlation Techniques for Full-Field Displacement Measurements of Micro Air Vehicle Flapping Wings", Experimental Techniques, November, 2009.

[18] LaVision, DaVis StrainMaster 3D, Software Package, Ver 7.2, Göttingen, Germany, January 2010.

[19] Mikhail, E., Bethel, J., McGlone, J., Introduction to Modern Photogrammetry, John Wiley and Sons, Inc., New York, 2001.

[20] Sicard, J., Sirohi, J., "Behavior of Extremely Flexible Rotor in Hover and Forward Flight", 66th AHS meeting, Phoenix, AZ, USA, 2010.

[21] Sicard, J., Sirohi, J., "Twist Control of an Extremely Flexible Rotor Blade for MicroAerial Vehicles", 67th AHS meeting, Tempe, AZ, USA, 2011.

[22] Sicard, J., "Thesis: Investigation of an Extremely Flexible Stowable Rotor for Micro-helicopters", University of Texas at Austin, May, 2011.

[23] Leishman, J.G., Principles of Helicopter Aerodynamics, $2^{\text {nd }}$ ed., Cambridge University Press, 2006.

[24] Hepperle, M., JavaFoil web-application, 2006. http://www.mh-aerotools.de/airfoils/javafoil.htm

[25] Drela, M., Xfoil application, Michigan Institute of Technology, 2001. http://web.mit.edu/drela/Public/web/xfoil/

[26] Knight, M. and Hefner, R.A., "Static Thrust of the Lifting Airscrew", NACA TN 626, 1937.

[27] Abbott, I.H.. and Von Doenhoff, A.E., "Theory of Wing Sections", Dover Publications, Inc., New York, 1959. 


\section{Vita}

Michael Skylar Lawson was born in Austin, Texas and grew up with strong physics and aeronautics influences from family as well as junior Air Force ROTC programs. Before pursuing higher education Michael aspired to a career in the military and served a short time in the Air Force before a medical discharge forced a career path change. He received his Bachelor of Science degree in Aerospace Engineering from the University of Texas in 2009.

Michael's graduate research interests include:

- Rotorcraft Design

- Optical Strain Measurement Techniques

- Robotics \& Automation

Permanent email address: mlawson.skylar@gmail.com

This thesis was typed by the author. 